\title{
Excision boundary conditions for black-hole initial data
}

\author{
Gregory B. Cook* \\ Department of Physics, Wake Forest University, Winston-Salem, North Carolina 27109, USA \\ Harald P. Pfeiffer ${ }^{\dagger}$ \\ Theoretical Astrophysics, California Institute of Technology, Pasadena, California 91125, USA
}

(Received 23 July 2004; published 12 November 2004)

\begin{abstract}
We define and extensively test a set of boundary conditions that can be applied at black-hole excision surfaces when the Hamiltonian and momentum constraints of general relativity are solved within the conformal thin-sandwich formalism. These boundary conditions have been designed to result in black holes that are in quasiequilibrium and are completely general in the sense that they can be applied with any conformal three-geometry and slicing condition. Furthermore, we show that they retain precisely the freedom to specify an arbitrary spin on each black hole. Interestingly, we have been unable to find a boundary condition on the lapse that can be derived from a quasiequilibrium condition. Rather, we find evidence that the lapse boundary condition is part of the initial temporal gauge choice. To test these boundary conditions, we have extensively explored the case of a single black hole and the case of a binary system of equal-mass black holes, including the computation of quasicircular orbits and the determination of the innermost stable circular orbit. Our tests show that the boundary conditions work well.
\end{abstract}

DOI: 10.1103/PhysRevD.70.104016

\section{INTRODUCTION}

The simulation of black-hole systems necessarily starts with the specification of initial data. In order for such simulations to yield astrophysically relevant results, the initial data must be constructed to be astrophysically realistic. Achieving this is the goal of efforts being made to improve black-hole, and in particular black-hole binary, initial data. It has become clear that all of the freely specifiable pieces of the initial data, including the boundary conditions (BC), must be chosen carefully to respect the physical content of any system we wish to simulate. In this paper we will focus on the boundary conditions that are required when a black hole's interior is excised from the initial-data domain.

In Ref. [1], one of the authors proposed a set of boundary conditions that were intended to yield a black hole that was in quasiequilibrium. These conditions were chosen to be consistent with the desire to create a binary system fully in quasiequilibrium. It is reasonable to expect such a system will be astrophysically realistic if the black holes in the binary are sufficiently far apart and in a nearly circular orbit. In this paper, we refine and extensively test these boundary conditions in the cases of a single black hole and a pair of equal-mass black holes in a binary system.

The most significant refinement of the quasiequilibrium boundary conditions over the original version in Ref. [1] is to the procedure for specifying the spin of each black hole. The analysis below shows that the spin must be

\footnotetext{
*Electronic address: cookgb@wfu.edu

${ }^{\dagger}$ Electronic address: harald@tapir.caltech.edu
}

PACS numbers: 04.25.Dm, 04.20.-q, 04.70.Bw, 97.80.-d

chosen in a very specific way in order to be compatible with the assumptions of quasiequilibrium. Fortunately, the procedure still allows for a completely arbitrary specification of the spin and this procedure is compatible with any choice of the conformal three-geometry.

A significant result from our tests on the original set of quasiequilibrium boundary conditions is that the proposed lapse boundary condition is not viable. We will show below that this boundary condition is degenerate when combined with the other quasiequilibrium boundary conditions. Furthermore, the nature of this degeneracy can be easily understood. We conjecture that the boundary condition on the lapse is not fixed by quasiequilibrium considerations but is, rather, a part of the initial temporal gauge choice. Below, we provide analytical and numerical evidence to support this conjecture.

For a single black hole, the quasiequilibrium boundary conditions allow for the construction of initial data that yield true stationary spacetimes. Doing so, however, requires that all of the freely specifiable data be chosen in a way that is compatible with a stationary black hole. In particular, it requires that the conformal three-geometry be chosen correctly. Unfortunately, there is still no general prescription for choosing an appropriate conformal three-geometry. Because of this, we have chosen to perform all of our tests on a flat conformal three-geometry. By testing the boundary conditions for the three cases of a single static, spinning, and boosted black hole, we will be able to test both the quasiequilibrium boundary conditions and the effect that the assumption of conformal flatness has on the resulting initial data. For equal-mass black hole binaries, we will extensively test the special cases of corotating and irrotational black holes. The nu- 
merical results we obtain will be compared with postNewtonian results and previous numerical results for both cases.

We begin in Sec. II with a review of the conformal thin-sandwich decomposition of the constraints, and then derive the quasiequilibrium boundary conditions in Sec. III. In Secs. IV and V we apply the boundary conditions to the cases of a single black hole and to equalmass black hole binary systems. Finally, in Sec. VI we further explore the effectiveness of these boundary conditions.

\section{THE CONFORMAL THIN-SANDWICH DECOMPOSITION}

In this work, we will use the standard $3+1$ decomposition with the interval written as

$$
\mathrm{d} s^{2}=-\alpha^{2} \mathrm{~d} t^{2}+\gamma_{i j}\left(\mathrm{~d} x^{i}+\beta^{i} \mathrm{~d} t\right)\left(\mathrm{d} x^{j}+\beta^{j} \mathrm{~d} t\right),
$$

where $\gamma_{i j}$ is the 3 -metric induced on a $t=$ const. spatial hypersurface, $\alpha$ is the lapse function, and $\beta^{i}$ is the shift vector. The extrinsic curvature of the spatial slice, $K_{i j}$, is defined by

$$
K_{\mu \nu} \equiv-\frac{1}{2} \gamma_{\mu}^{\delta} \gamma_{\nu}^{\rho} \mathcal{L}_{n} \gamma_{\delta \rho},
$$

where $\mathcal{L}_{n}$ denotes the Lie derivative along the unit normal to the spatial slice, $n^{\mu}$. Einstein's equations, in vacuum, then reduce to four sets of equations. Two are evolution equations for the spatial metric and extrinsic curvature:

$$
\partial_{t} \gamma_{i j}=-2 \alpha K_{i j}+2 \bar{\nabla}_{(i} \beta_{j)},
$$

and

$$
\begin{aligned}
\partial_{t} K_{i j} & =-\bar{\nabla}_{i} \bar{\nabla}_{j} \alpha+\alpha\left[\bar{R}_{i j}-2 K_{i \ell} K_{j}^{\ell}+K K_{i j}\right] \\
& +\beta^{\ell} \bar{\nabla}_{\ell} K_{i j}+2 K_{\ell(i} \bar{\nabla}_{j)} \beta^{\ell} .
\end{aligned}
$$

The remaining two are the constraint equations

$$
\bar{R}+K^{2}-K_{i j} K^{i j}=0
$$

and

$$
\bar{\nabla}_{j}\left(K^{i j}-\gamma^{i j} K\right)=0 .
$$

Here, $\bar{\nabla}_{i}, \bar{R}_{i j}$, and $\bar{R}$ are, respectively, the covariant derivative, Ricci tensor, and Ricci scalar associated with the spatial metric $\gamma_{i j}$. Finally, the trace of the extrinsic curvature is denoted $K \equiv K_{i}^{i}$.

The task of constructing initial data for a Cauchy evolution via Einstein's equations requires that we decompose the constraints in such a way that we can specify how the constrained, gauge, and dynamical degrees of freedom are associated with the initial data. In this paper, we are primarily interested in initial data associated with systems in quasiequilibrium. Because of this, it is natural to use the conformal thin-sandwich decomposition of the constraints $[2,3]$. This decomposition is particularly useful in this situation because quasiequilibrium is a dynamical concept, and this decomposition retains a close connection to dynamics that is lost in most other decompositions of the constraints (cf. Refs. [1,4]).

The conformal thin-sandwich decomposition employs a York-Lichnerowicz conformal decomposition of the metric and various other quantities [5-7]. The conformal factor $\psi$ is defined via

$$
\gamma_{i j} \equiv \psi^{4} \tilde{\gamma}_{i j}
$$

where $\tilde{\gamma}_{i j}$ is a "conformal metric." The time derivative of the conformal metric is introduced by the definitions

$$
\begin{aligned}
\tilde{u}_{i j} & \equiv \partial_{t} \tilde{\gamma}_{i j}, \\
\tilde{\gamma}^{i j} \tilde{u}_{i j} & \equiv 0 .
\end{aligned}
$$

From this, it follows that the trace-free extrinsic curvature $A^{i j} \equiv K^{i j}-\frac{1}{3} \gamma^{i j} K$ takes the form

$$
A^{i j}=\frac{\psi^{-10}}{2 \tilde{\alpha}}\left[(\tilde{\mathbb{L}} \beta)^{i j}-\tilde{u}^{i j}\right],
$$

where $\tilde{\alpha} \equiv \psi^{-6} \alpha$ is the conformal lapse function, and $\tilde{u}^{i j}=\tilde{u}_{k l} \tilde{\gamma}^{i k} \tilde{\gamma}^{j l}$. Furthermore, $(\tilde{\mathbb{L}} V)$ is the conformal Killing (or longitudinal) operator acting on a vector, defined by

$$
(\tilde{\mathbb{Z}} V)_{i j} \equiv 2 \tilde{\nabla}_{(i} V_{j)}-\frac{2}{3} \tilde{\gamma}_{i j} \tilde{\nabla}_{k} V^{k}
$$

where $\tilde{\nabla}_{k}$ is the covariant derivative compatible with $\tilde{\gamma}_{i j}$.

Notice that this decomposition of $K_{i j}$ incorporates the kinematical variables of the $3+1$ decomposition, that is, the shift vector $\beta^{i}$ and the lapse function $\alpha$ through the conformal lapse $\tilde{\alpha}$. It also includes the trace-free time derivative of the conformal metric, $\tilde{u}_{i j}$. Below, the conformal trace-free extrinsic curvature will be useful,

$$
\tilde{A}^{i j} \equiv \psi^{10} A^{i j}=\frac{1}{2 \tilde{\alpha}}\left[(\tilde{\mathbb{L}} \beta)^{i j}-\tilde{u}^{i j}\right] .
$$

Within the conformal thin-sandwich formalism, one must specify $\tilde{\gamma}_{i j}, \tilde{u}_{i j}, K$, and $\tilde{\alpha}$. With these quantities defined, the Hamiltonian (5) and momentum (6) constraints take the form of a coupled set of elliptic equations that determine $\psi$ and $\beta^{i}$. In terms of our conformally decomposed variables, the Hamiltonian constraint (5) can be written

$$
\tilde{\nabla}^{2} \psi-\frac{1}{8} \psi \tilde{R}-\frac{1}{12} \psi^{5} K^{2}+\frac{1}{8} \psi^{-7} \tilde{A}_{i j} \tilde{A}^{i j}=0,
$$

where $\tilde{R}$ is the Ricci scalar associated with $\tilde{\gamma}_{i j}$, and the momentum constraint (6) as

$$
\tilde{\nabla}_{j}\left[\frac{1}{2 \tilde{\alpha}}(\tilde{\mathbb{L}} \beta)^{i j}\right]-\frac{2}{3} \psi^{6} \tilde{\nabla}^{i} K-\tilde{\nabla}_{j}\left(\frac{1}{2 \tilde{\alpha}} \tilde{u}^{i j}\right)=0 .
$$


The freely specified data includes the conformal metric $\tilde{\gamma}_{i j}$ and its time derivative $\tilde{u}_{i j}=\partial_{t} \tilde{\gamma}_{i j}$, as well as the trace of the extrinsic curvature, $K$, and the conformal lapse $\tilde{\alpha}$. It is possible, and desirable, to make the set of freely specified data more symmetric by choosing to specify the time derivative of the extrinsic curvature instead of the conformal lapse. This is possible because these two quantities are related by the trace of Eq. (4). The resulting equation is an elliptic equation for the conformal lapse that is coupled to both the Hamiltonian and momentum constraints, (13) and (14). This equation can be written as

$$
\begin{aligned}
& \tilde{\nabla}^{2}\left(\psi^{7} \tilde{\alpha}\right)-\left(\psi^{7} \tilde{\alpha}\right)\left(\frac{1}{8} \tilde{R}+\frac{5}{12} \psi^{4} K^{2}+\frac{7}{8} \psi^{-8} \tilde{A}_{i j} \tilde{A}^{i j}\right) \\
& \quad=-\psi^{5}\left(\partial_{t} K-\beta^{k} \tilde{\nabla}_{k} K\right) .
\end{aligned}
$$

The statement made earlier, that the conformal thinsandwich decomposition has a close connection to dynamics, is now clear. Not only does this decomposition incorporate the kinematical variables of the $3+1$ splitting, but fully half of the freely specifiable data consist of time derivatives of fundamental fields. In particular, we are free to choose the conformal metric and the trace of the extrinsic curvature $\left(\tilde{\gamma}_{i j}\right.$ and $\left.K\right)$ and the time derivatives of these fields $\left(\partial_{t} \tilde{\gamma}_{i j} \equiv \tilde{u}_{i j}\right.$ and $\left.\partial_{t} K\right)$.

Remaining to be determined are the conformal factor $\psi$, the conformal lapse $\tilde{\alpha}$, and the shift vector $\beta^{i}$. These are determined by solving Eqs. (13)-(15) as a coupled set of elliptic equations. Formulating a well-posed system requires that we impose boundary conditions. Typically, these systems are solved under the assumption that the spacetime is asymptotically flat. If we let $r$ denote a coordinate radius measured from the location of the center of energy of the system, then as $r \rightarrow \infty$ we have that

$$
\begin{aligned}
\left.\psi\right|_{r \rightarrow \infty} & =1, \\
\left.\beta^{i}\right|_{r \rightarrow \infty} & =\left(\Omega_{0} \times \mathbf{r}\right)^{i}, \\
\left.\alpha\right|_{r \rightarrow \infty}=\left.\tilde{\alpha}\right|_{r \rightarrow \infty} & =1 .
\end{aligned}
$$

$\Omega_{0}$ is the orbital angular velocity of a binary system, or the rotational angular velocity of a single object, as measured at infinity. The boundary condition on the shift is chosen so that the time coordinate, $t^{\mu}=\alpha n^{\mu}+\beta^{\mu}$, is helical and tracks the rotation of the system $[1,8,9]$. If we wish to consider systems with one or more black holes, and if we excise the interior of the black hole to avoid difficulties with singularities, then we will also need to impose boundary conditions on the excision surfaces. This is the topic of the next section.

\section{BLACK-HOLE EXCISION BOUNDARY CONDITIONS}

The physical content of initial data depends on the choices made for the initial-data decomposition scheme, freely specifiable data, and the boundary conditions.
Therefore, it is important to choose boundary conditions that are motivated by, or at least compatible with, the sort of initial data that we wish to construct.

The first attempts to impose boundary conditions on black-hole excision boundaries were based on topological arguments [10-13]. By demanding that the initial-data hypersurface consist of two identical (isometric) asymptotically flat hypersurfaces connected together at a number of spherical excision surfaces (one for each black hole), it is possible to show that the surfaces where the isometric sheets connect are fixed point sets of the isometry. This condition is enough to determine either Dirichlet or Neumann boundary conditions at the excision surface for any fields that are present.

Boundary conditions based on this idea have been used successfully for generating general black-hole initial data using various initial-data decompositions [14-17]. Their first use in conjunction with the conformal thin-sandwich decomposition $[9,18]$ was only partially successful due to an unavoidable constraint violation. The difficulties with this approach were outlined in Ref. [1], where an alternative approach of using quasiequilibrium boundary conditions was first outlined. In this section, we will refine, and in subsequent sections test, this approach.

In constructing initial data on a spacelike hypersurface, we cannot have knowledge of the event horizon that is typically used to define the surface of a black hole. However, we can identify the apparent horizon of a black hole, defined as the outermost marginally outer-trapped surface. A marginally outer-trapped surface (MOTS), in turn, is a surface on which the expansion $\theta$ of the family of outgoing null rays, $k^{\mu}$, vanishes everywhere.

In this paper, we are interested in the situation in which each black hole is in quasiequilibrium. The assumptions required to enforce this are essentially the same as those required of an "isolated horizon" (cf. [19-21]). To ensure that the black hole is in quasiequilibrium, we enforce the following conditions. First, we demand that the expansion $\theta$ vanish on the excision surface $S$, thus forcing the boundary to be an apparent horizon:

$$
\left.\theta\right|_{S}=0 .
$$

Next, we require that the shear $\sigma_{\mu \nu}$ of the outgoing null rays also vanish on the excision boundary,

$$
\left.\sigma_{\mu \nu}\right|_{S}=0 .
$$

Consider the family of null geodesics threading the apparent horizon to the future of our initial-data slice that are tangent to $k^{\mu}$ on $S$. Raychaudhuri's equation for null congruences,

$$
\mathcal{L}_{k} \theta=-\frac{1}{2} \theta^{2}-\sigma_{\mu \nu} \sigma^{\mu \nu}+\omega_{\mu \nu} \omega^{\mu \nu}-R_{\mu \nu} k^{\mu} k^{\nu},
$$

together with Eqs. (17) and (18) are sufficient to imply that 


$$
\left.\mathcal{L}_{k} \theta\right|_{S}=0 .
$$

That is, initially, the apparent horizon will evolve along $k^{\mu}$. Note that $\omega_{\mu \nu}$ is the twist of the congruence, which vanishes because the congruence is surface forming. Also, we assume that there is no matter on $S$, so $R_{\mu \nu}=0$.

While conditions (17)-(19) are coordinate independent, our next and final demand breaks precisely this coordinate freedom: We demand that the coordinate location of the apparent horizon does not move initially in an evolution of the initial data.

As we show in the subsequent sections, the requirements listed so far yield four conditions that can be imposed on the initial data at the excision boundary. However, there are five coupled initial-data equations that must be solved in the conformal thin-sandwich approach. When quasiequilibrium black-hole boundary conditions were first derived, a fifth condition was considered [1]. In particular, the condition that $\mathcal{L}_{k} \dot{\theta}=0$ was considered, where $\theta$ is the expansion of a family of ingoing null rays, $k^{\mu}$. As we will show below, this fifth condition cannot be used as a boundary condition even though it is satisfied for a stationary black hole!

In the remainder of this section, we will derive boundary conditions for black-hole excision surfaces based on the demands outlined above. A good portion of the following derivation appeared previously [1]. However, because of a change in notation, and more importantly in a few sign conventions, we include the full derivation below.

\section{A. Geometry of the Excision Boundary}

We demand that the excision boundary surface $S$ be a spacelike 2-surface with topology $S^{2}$ and define $s^{i}$ to be the outward pointing unit vector normal to the surface. In this case, we define outward with respect to the black hole (not the domain), so that $s^{i}$ points toward infinity. The four-dimensional generalization of $s^{i}$ has components $s^{\mu}=\left[0, s^{i}\right]$ obtained from the condition that $s^{\mu} n_{\mu}=0$.

The metric $h_{i j}$ induced on $S$ by $\gamma_{i j}$ is given by

$$
h_{i j} \equiv \gamma_{i j}-s_{i} s_{j}
$$

We also define the extrinsic curvature $H_{i j}$ of $S$ embedded in the three-dimensional spatial hypersurface as

$$
H_{i j} \equiv h_{i}^{k} h_{j}^{\ell} \bar{\nabla}_{(k} s_{\ell)}=\frac{1}{2} h_{i}^{k} h_{j}^{\ell} \mathcal{L}_{s} h_{k \ell} .
$$

Naturally associated with $S$ are two sets of null vectors: a set of outgoing null rays, $k^{\mu}$, and a set of ingoing null rays, $k^{\mu}$, defined by

$$
k^{\mu} \equiv \frac{1}{\sqrt{2}}\left(n^{\mu}+s^{\mu}\right) \quad \text { and } \quad \dot{k}^{\mu} \equiv \frac{1}{\sqrt{2}}\left(n^{\mu}-s^{\mu}\right) .
$$

Associated with each set of null rays is an extrinsic curvature of $S$ as embedded in the full four-dimensional manifold. These are defined as

$$
\begin{aligned}
& \Sigma_{\mu \nu} \equiv \frac{1}{2} h_{\mu}^{\alpha} h_{\nu}^{\beta} \mathcal{L}_{k} g_{\alpha \beta}, \\
& \dot{\Sigma}_{\mu \nu} \equiv \frac{1}{2} h_{\mu}^{\alpha} h_{\nu}^{\beta} \mathcal{L}_{k} g_{\alpha \beta},
\end{aligned}
$$

where $g_{\mu \nu}$ is the full spacetime metric. Because these tensors $\Sigma_{\mu \nu}$ and $\dot{\Sigma}_{\mu \nu}$ are spatial, we will use spatial indices below. To simplify the definitions that follow, we will introduce various projections of $K_{i j}$ along and normal to the excision boundary $S$ :

$$
\begin{gathered}
J_{i j} \equiv h_{i}^{k} h_{j}^{\ell} K_{k \ell}, \\
J_{i} \equiv h_{i}^{k} s^{\ell} K_{k \ell}, \\
J \equiv h^{i j} J_{i j}=h^{i j} K_{i j} .
\end{gathered}
$$

We can then simplify Eqs. (24) and (25) to

$$
\Sigma_{i j}=-\frac{1}{\sqrt{2}}\left(J_{i j}-H_{i j}\right) \quad \text { and } \quad \dot{\Sigma}_{i j}=-\frac{1}{\sqrt{2}}\left(J_{i j}+H_{i j}\right) \text {. }
$$

Now, we define the expansion of outgoing null rays, $\theta$, and ingoing null rays, $\hat{\theta}$, via

$$
\begin{gathered}
\theta \equiv h^{i j} \sum_{i j}=-\frac{1}{\sqrt{2}}(J-H), \\
\dot{\theta} \equiv h^{i j} \dot{\Sigma}_{i j}=-\frac{1}{\sqrt{2}}(J+H) .
\end{gathered}
$$

Finally, we define the shear of the outgoing null rays, $\sigma_{i j}$, and ingoing null rays, $\sigma_{i j}$, via

$$
\begin{gathered}
\sigma_{i j} \equiv \Sigma_{i j}-\frac{1}{2} h_{i j} \theta, \\
\hat{\sigma}_{i j} \equiv \dot{\Sigma}_{i j}-\frac{1}{2} h_{i j} \dot{\theta} .
\end{gathered}
$$

\section{B. Quasiequilibrium Boundary Conditions}

With the definitions of Sec. III A, we can now evaluate the demands we made earlier in Sec. III and translate them into boundary conditions for the conformal thinsandwich equations. In order to express these as useful boundary conditions, we must write them in terms of the variables of the conformal thin-sandwich approach. We must also make connection with the global notion of quasiequilibrium that is closely associated with an approximate helical Killing vector.

A spacetime that is in true equilibrium is said to be stationary and has two Killing vectors of interest: a timelike Killing vector, $\partial / \partial t_{0}$, and a spatial Killing vector 
associated with rotational symmetry, $\partial / \partial \phi_{0}$. If $\Omega_{0}$ denotes the angular velocity of a spinning object or system as measured at infinity, then the linear combination $\partial / \partial t_{0}+\Omega_{0} \partial / \partial \phi_{0}$ is referred to as the helical Killing vector. If a system, such as a binary, is in a state of quasiequilibrium, there are in general no vector fields similar to $\partial / \partial t_{0}$ or $\partial / \partial \phi_{0}$ that are even close to being Killing vectors. But there will be a helical vector field that is an approximate Killing vector of the spacetime. If we let this approximate Killing vector field define our time vector $t^{\mu}$, and thus our time coordinate $t$, then we will have $\partial / \partial t \approx 0$ for fields in this spacetime. Within the $3+1$ decomposition, we write the time vector as

$$
t^{\mu}=\alpha n^{\mu}+\beta^{\mu} .
$$

Our desire for $t^{\mu}$ to represent an approximate helical Killing vector is, therefore, the reason for our condition on the shift at infinity, Eq. (16b).

Now we consider the demand that the apparent horizon should initially not move in an evolution of the quasiequilibrium initial data. Because of Eq. (20), the apparent horizon initially coincides with the null surface generated by $k^{\mu}$. In order for the coordinates to track this null surface, the time vector of the evolution, $t^{\mu}$, must lie in this null surface. This requires that

$$
\left.t^{\mu} k_{\mu}\right|_{S}=0 \text {. }
$$

Substituting Eqs. (23) and (34) into Eq. (35), and recalling that the shift vector is spatial, $\beta^{\mu} n_{\mu}=0$, yields

$$
\left.\alpha\right|_{S}=\left.\beta^{i} s_{i}\right|_{S}
$$

This equation is often referred to as the Killing-horizon condition. We split the shift vector into its component normal to the surface, $\beta_{\perp}$, and a vector tangent to the surface, $\beta_{\|}^{i}$, defined by

$$
\begin{aligned}
& \beta_{\perp} \equiv \beta^{i} s_{i}, \\
& \beta_{\|}^{i} \equiv h_{j}^{i} \beta^{j} .
\end{aligned}
$$

With these definitions, we see that Eq. (36) is a condition on the normal component of the shift,

$$
\left.\beta_{\perp}\right|_{S}=\left.\alpha\right|_{S} .
$$

The component of the shift tangential to the excision surface $S, \beta_{\|}^{i}$, remains unconstrained so far. This makes sense, because fixing the location of a surface does not restrict motion within this surface. We can gain insight into the relevance of $\beta_{\|}^{i}$ by considering a stationary Kerr black hole.

The Kerr spacetime has two Killing vectors of interest: a timelike Killing vector, $\partial / \partial t_{0}$, and a spatial Killing vector associated with rotational symmetry, $\partial / \partial \phi_{0}$. The null generators of the horizon are given by

$$
k=\frac{\partial}{\partial t_{0}}+\Omega_{H} \frac{\partial}{\partial \phi_{0}},
$$

where $\Omega_{H}$ is the angular frequency of the horizon. If we introduce a fiducial helical Killing vector,

$$
\ell=\frac{\partial}{\partial t_{0}}+\Omega \frac{\partial}{\partial \phi_{0}},
$$

for some $\Omega$, then, of course, on the horizon,

$$
\ell=k+\left(\Omega-\Omega_{H}\right) \frac{\partial}{\partial \phi_{0}} .
$$

Now consider a hypersurface through Kerr to which $\partial / \partial \phi_{0}$ is always tangent, e.g., the usual Kerr-Schild slice. To make the connection with the usual $3+1$ decomposition straightforward, we normalize $k$ and $\ell$ by choosing their time components to be one $\left(k^{t}=\ell^{t}=1\right)$. If we choose the vector $\ell$ as the time vector of an evolution, then the last term in Eq. (42) corresponds precisely to $\beta_{\|}^{i}$-this term is tangent both to the horizon and to the hypersurface. For the choice $\beta_{\|}^{i}=0$, the last term in Eq. (42) would be absent, i.e., $\Omega=\Omega_{H}$. In this case the black hole is corotating with the coordinate system, and the generators of the horizon, $k^{\mu}$ do not twist relative to the helical Killing vector $\ell^{\mu}$. Conversely, a nonrotating black hole $\left(\Omega_{H}=0\right)$ would have a tangential shift of

$$
\beta_{\|}^{i}=\Omega\left(\frac{\partial}{\partial \phi_{0}}\right)^{i}
$$

For a binary black hole in quasiequilibrium, neither $\partial / \partial t_{0}$ nor $\partial / \partial \phi_{0}$ exist as separate Killing vectors. However, based on the discussion above, we expect that $\beta_{\|}^{i}$ is still connected to the rotation of the black hole. In the case $\beta_{\|}^{i}=0$, the horizon generators have no twist relative to the helical Killing vector (which coincides with the time vector), corresponding to corotating black holes. Any rotation, $\beta_{\|}^{i} \propto\left(\partial / \partial \phi_{S}\right)^{i}$, where $\left(\partial / \partial \phi_{S}\right)^{i}$ lies in the surface $S$ would impart additional rotation on the black hole. Below we will make these notions precise.

Having obtained a boundary condition on $\beta_{\perp}$, we now turn our attention to Eqs. (17) and (18). We need to consider the horizon boundary $S$ in the conformal space. The conformal transformation on $\gamma_{i j}$ (7) induces a natural conformal weighting for $h_{i j}$ and for the unit normal to $S$,

$$
\begin{aligned}
& h_{i j} \equiv \psi^{4} \tilde{h}_{i j}, \\
& s^{i} \equiv \psi^{-2} \tilde{s}^{i} .
\end{aligned}
$$

If we also define $\tilde{D}^{i}$ as the covariant derivative compatible with $\tilde{h}_{i j}$, then without loss of generality, we can express the expansion of the outgoing null rays, $\theta$, as

$$
\theta=\frac{\psi^{-2}}{\sqrt{2}}\left(\tilde{h}^{i j} \tilde{\nabla}_{i} \tilde{s}_{j}+4 \tilde{s}^{k} \tilde{\nabla}_{k} \ln \psi-\psi^{2} J\right),
$$


and the shear of the outgoing null rays, $\sigma^{i j}$, as

$$
\begin{aligned}
\sigma^{i j}= & \frac{1}{\sqrt{2}}\left(H^{i j}-\frac{1}{2} h^{i j} H\right)\left(1-\frac{\beta_{\perp}}{\alpha}\right)-\frac{1}{\sqrt{2}} \frac{\psi^{-4}}{\alpha} \\
& \times\left[\tilde{D}^{(i} \beta_{\|}^{j)}-\frac{1}{2} \tilde{h}^{i j} \tilde{D}_{k} \beta_{\|}^{k}-\frac{1}{2}\left(\tilde{h}_{k}^{i} \tilde{h}_{\ell}^{j} \tilde{u}^{k \ell}-\frac{1}{2} \tilde{h}^{i j} \tilde{h}_{k \ell} \tilde{u}^{k \ell}\right)\right] .
\end{aligned}
$$

It is now clear how to obtain the remaining boundary conditions. By applying condition (17) to Eq. (46), we obtain a boundary condition that forces an excision boundary to be an apparent horizon (or MOTS). The condition is

$$
\left.\tilde{s}^{k} \tilde{\nabla}_{k} \ln \psi\right|_{S}=-\left.\frac{1}{4}\left(\tilde{h}^{i j} \tilde{\nabla}_{i} \tilde{s}_{j}-\psi^{2} J\right)\right|_{S},
$$

and it takes the form of a nonlinear Robin-type boundary condition on the conformal factor, $\psi$. Finally, if we recall the condition from Eq. (39) and that we have chosen $\tilde{u}^{i j}=$ 0 everywhere, and apply condition (18) to Eq. (47), we obtain a condition that restricts the form of $\beta_{\|}^{i}$. The condition is that

$$
\left.\tilde{D}^{(i} \beta_{\|}^{j)}\right|_{S}-\left.\frac{1}{2} \tilde{h}^{i j} \tilde{D}_{k} \beta_{\|}^{k}\right|_{S}=0 .
$$

This shows that the components of the shift that are associated with the spin of the black hole must be proportional to a conformal Killing vector of the conformal metric, $\tilde{h}_{i j}$, defined on the two-dimensional excision surface.

This condition is quite remarkable. Recall that any two-surface that is topologically $S^{2}$ is conformally equivalent to the unit two-sphere. If you consider a unit two-sphere embedded in a flat three-dimensional Euclidean space, then there is a family of rotational Killing vectors, $\xi^{i}$, associated with any rotation axis passing through the center of the two-sphere. Because $\xi^{i} \hat{n}_{i}=0$ on the two-sphere, where $\hat{n}^{i}$ is the unit normal vector on the two-sphere, we see that $\xi^{i}$ trivially form a family of two-dimensional vectors tangent to the twosphere and that these are Killing vectors of the metric on the unit two-sphere. But, the Killing vectors associated with any metric are also conformal Killing vectors of any metric conformally related to it. So, if $\varphi$ represents a conformal transformation such that $\varphi^{4} \tilde{h}_{i j}$ is the metric of the unit two-sphere, then $\xi^{i}$ will satisfy the conformal Killing equation on $\tilde{h}_{i j}$. Thus,

$$
\beta_{\|}^{i}=\Omega_{r} \xi^{i}
$$

will satisfy Eq. (49), with $\Omega_{r}$ being an arbitrary parameter. The freedom left in Eq. (50) is precisely what is required to parametrize an arbitrary spin on the black hole. The parameter $\Omega_{r}$ is associated with the magnitude of the rotation or spin of the black hole, whereas the axis of rotation of $\xi^{i}$ is related to the orientation of the spin. Of course, $\Omega_{r}$ does not correspond directly to the rotational angular velocity of the black hole. From the discussion leading to Eq. (43), it is clear that $\Omega_{r}=0$ corresponds to a black hole that is corotating with an approximate helical Killing vector $t^{\mu}$ and thus represents a black hole that is rotating as seen by an inertial observer at infinity. In order to construct a black hole that is not rotating as seen from infinity, we need to choose a shift that is similar in form to Eq. (43). It seems reasonable to choose $\Omega_{r}=\Omega_{0}$ and to pick the conformal Killing vector $\xi^{i}$ from the family that corresponds to rotation about an axis that is perpendicular to the plane of the orbit.

To summarize, the quasiequilibrium conditions defined in Eqs. (17) and (18) define boundary conditions on the conformal factor, $\psi$, via Eq. (48) and on the shift vector, $\beta^{i}$, via Eqs. (37)-(39) and (50). These total to four of the five necessary boundary conditions for solving the coupled elliptic equations associated with the conformal thin-sandwich equations. Missing is a condition on the conformal lapse, $\tilde{\alpha}$.

\section{Boundary Conditions on the Lapse Function}

A possible boundary condition on the lapse was derived in Ref. [1]. The condition was essentially based on the reasonable quasiequilibrium condition that $\mathcal{L}_{k} \dot{\theta}=0$. Notice that this is the change in the expansion of ingoing null rays, $k^{\mu}$, with respect to the outgoing null congruence. The resulting boundary condition takes the form

$$
\left.\alpha_{B C} \equiv\left(J \tilde{s}^{i} \tilde{\nabla}_{i} \alpha-\mathcal{E} \alpha\right)\right|_{S}=0,
$$

where $\mathcal{E}$ is a nonlinear operator that is elliptic within the surface $S$ (see Eq. (84) of Ref. [1] for a precise description). This condition is satisfied on the horizon of a stationary black hole and it seems to supply a reasonable boundary condition for the lapse to be used in conjunction with the previously defined boundary conditions for the conformal factor and shift vector.

We implemented the full set of boundary conditions within the code described in Ref. [22] and attempted to solve the full set of conformal thin-sandwich equations for the case of a single non rotating black hole. It became clear immediately that the iterative solutions would not converge in general. Interestingly, if the analytic solution for an isolated black hole was supplied for the starting point of the iterations, then the equations and boundary conditions were satisfied to truncation error, but the iterations were at best only marginally stable. Furthermore, if any one of the boundary conditions on $\psi, \beta_{\perp}$, or $\alpha$ were replaced by an arbitrary Dirichlet or Neumann boundary condition, then the iterative solution was convergent to a solution representing a static black hole and the omitted boundary condition was satisfied.

This clearly indicates that the set of boundary conditions including Eq. (51) is degenerate and leads to an illposed elliptic system. We can understand the nature of the degeneracy by considering the family of time- 
independent maximal slicings of Schwarzschild [23-25]. The line element for the spatial metric, lapse, and shift vector are

$$
\begin{aligned}
\mathrm{d} s^{2} & =\left(1-\frac{2 M}{R}+\frac{C^{2}}{R^{4}}\right)^{-1} \mathrm{~d} R^{2}+R^{2} \mathrm{~d} \Omega^{2}, \\
\alpha & =\sqrt{1-\frac{2 M}{R}+\frac{C^{2}}{R^{4}}} \\
\beta^{R} & =\frac{C}{R^{2}} \sqrt{1-\frac{2 M}{R}+\frac{C^{2}}{R^{4}}},
\end{aligned}
$$

where $R$ is the usual Schwarzschild "areal" radial coordinate, $M$ is the mass of the black hole, and $C$ is a constant parametrizing the family of maximal slicings. In spherical coordinates, the extrinsic curvature takes the form

$$
K_{j}^{i}=\frac{C}{R^{3}}\left[\begin{array}{ccc}
-2 & 0 & 0 \\
0 & 1 & 0 \\
0 & 0 & 1
\end{array}\right] .
$$

For $0 \leq C / M^{2}<\sqrt{\frac{27}{16}} \approx 1.299$ the maximal spatial slice extends from spatial infinity, through the black-hole interior, and to the second spatial infinity of the maximally extended Schwarzschild geometry. When $C / M^{2}=0$, we recover the standard Schwarzschild maximal slice that passes through the bifurcation point. And, for $C / M^{2}>$ $\sqrt{\frac{27}{16}}$, the maximal spatial slice extends from spatial infinity, through the black-hole horizon, and ends on the singularity.

If desired, the family of maximal slicings of Schwarzschild can be rewritten in terms of an isotropic radial coordinate, $r$. It is then easy to verify that the boundary conditions (39), (48), and (51) are satisfied on the horizon for any value of $C / M^{2}$. So, we see that while the set of boundary conditions proposed in Ref. [1] hold for a time-independent configuration, they do not uniquely fix the spatial slicing. It is in this way that they are degenerate.

If we consider the value of the lapse on the horizon, we find that

$$
\alpha\left(r_{\mathcal{H}}\right)=\frac{1}{4}\left(\frac{C}{M^{2}}\right),
$$

where $r_{\mathcal{H}}$ denotes the location of the horizon in isotropic coordinates. Note that $r_{\mathcal{H}} \neq M / 2$ unless $C / M^{2}=0$. If, instead of using the lapse boundary condition (51), we simply fix a Dirichlet value for the lapse, then we find that we have effectively chosen a value of $C / M^{2}$ and thus a particular maximal slicing of Schwarzschild. Similarly, we find that the family of mixed boundary conditions

$$
\left.\frac{\partial \alpha}{\partial r}\right|_{r_{\mathcal{H}}}=\left.\mathcal{A} \frac{\alpha}{r}\right|_{r_{\mathcal{H}}}
$$

corresponds to a slicing choice of $C / M^{2}=\sqrt{\mathcal{A}^{2}+4}-$
$\mathcal{A}$, where $\mathcal{A}$ is any real number. It is clear that any reasonable choice of a Dirichlet, Neumann, or mixed boundary condition on the lapse will uniquely fix a particular maximal slicing of Schwarzschild and effectively break the degeneracy.

As mentioned previously, our numerical investigations have shown that we could also have chosen to use the lapse boundary condition (51) and instead fix either $\psi$ or $\beta_{\perp}$ on the horizon via a Dirichlet, Neumann, or mixed boundary condition. One reason to choose to replace the lapse condition (51), as opposed to the boundary conditions on the conformal factor (48) or the shift (39), is that the lapse boundary condition is much more complex. However, there is a more fundamental reason to choose to replace the lapse condition. The degeneracy that we must eliminate is in the choice of the spatial slice which is a choice of the initial temporal gauge. The lapse function fixes the evolution of the temporal gauge. Therefore it seems reasonable that we should consider the choice of the lapse boundary condition as part of the initial temporal gauge choice. It is customary to view the choice of the trace of the extrinsic curvature as fixing the initial temporal gauge. However, this is apparently not sufficient within the conformal thin-sandwich approach when interior boundaries are present.

This last assertion, that the lapse boundary condition must be chosen as part of the initial temporal gauge choice, is supported by the behavior of a very special solution of Einstein's equations - the static Schwarzschild solution. However the same generic behavior is seen in a broad class of examples as we will outline below.

\section{QUASIEQUILIBRIUM SOLUTIONS FOR A SINGLE BLACK HOLE}

As we have seen in Sec. II, the conformal thinsandwich equations require specification of free data, which are the conformal metric $\tilde{\gamma}_{i j}$ and its time derivative $\tilde{u}_{i j}$, as well as the mean curvature $K$ and its time derivative $\partial_{t} K$. Moreover, boundary conditions are required on the variables being solved for, the conformal factor $\psi$, the shift $\beta^{i}$, and the lapse $\alpha$. The quasiequilibrium approximation fixes a good portion of these choices, namely $\tilde{u}_{i j}=\partial_{t} K=0$, as well as the following boundary conditions at the excised regions: The apparent horizon condition Eq. (48) on $\psi$, the null horizon condition Eq. (39) on $\beta_{\perp}$, and Eq. (50) on $\beta_{\|}^{i}$. At spatial infinity, the boundary conditions are straightforward and are given by Eqs. (16).

Before the conformal thin-sandwich equations can be solved, we have to choose the remaining quantities which are not fixed by the quasiequilibrium framework. These are $\tilde{\gamma}_{i j}, K$, and an inner boundary condition on the lapse $\alpha$. Furthermore, we have to choose the shape of the 
excised regions, $S$. As argued above, and as we confirm below, the lapse boundary condition and $K$ are part of the temporal gauge choice. It thus remains to choose $\tilde{\gamma}_{i j}$ and the shape of the excised regions, $S$.

In this work, we will assume that the conformal threegeometry is flat, and we will always excise exact spheres. These choices are not motivated by physical considerations, and they will affect the quality of the quasiequilibrium solutions we obtain in this paper. For example, the Kerr spacetime does not admit conformally flat slices $[26,27]$. Therefore, when we solve for a rotating black hole, our initial-data sets will not exactly represent a Kerr black hole, but will rather correspond to a perturbed Kerr black hole, which will settle down to Kerr. We stress that this failure of the initial-data sets constructed here to represent Kerr is not inherent in the quasiequilibrium method, but is caused by our choices for $\tilde{\gamma}_{i j}$ and $S$. With the appropriate choices for $\tilde{\gamma}_{i j}$ and $S$, the quasiequilibrium method can reproduce exactly any timeindependent solution of Einstein's equations. Indeed, for single black holes, better choices for $\tilde{\gamma}_{i j}$ and $S$ are easily obtained from stationary analytic solutions of Einstein's equations, for example, based on Kerr-Schild coordinates. While such a choice certainly leads to single black hole initial-data sets closer to the true Kerr metric, it is not clear how to generalize to binary black-hole configurations. A widely used approach superposes single blackhole quantities to construct binary black- hole initial data (e.g., [16,28-31]). However, because of the nonlinear nature of Einstein's equations, and since often the black holes are separated by only a few Schwarzschild radii, the superposition introduces uncertainties that may be large [22] and that have not yet been adequately quantified. In this paper, rather than using superposition, we start with choices for $\tilde{\gamma}_{i j}$ and $S$ that are not optimal for single blackhole spacetimes, but that are equally well suited for binary black-hole configurations. We then use the single black-hole solutions to quantify the effects of our approximation.

We solve the conformal thin-sandwich equations with the pseudospectral collocation method described in [32]. For the single black-hole spacetimes, typically, two spherical shells are employed. The inner one ranges from the excised sphere to a radius of $\sim 20$ and distributes grid-points exponentially in radius. The outer shell has an outer radius of typically $\sim 10^{10}$, and employs an inverse mapping in radius, which is well adapted to the $1 / r$-falloff of many quantities. The fifth elliptic equation (for the lapse function) is coded in the form of Eq. (15), i.e., as an equation for $\psi^{7} \tilde{\alpha}=\alpha \psi$. Therefore, we formulate the lapse boundary condition as a condition on $\alpha \psi$. Finally, we note that we always solve the threedimensional initial value equations, even in cases which have spherical or cylindrical symmetries like the single black-hole solutions.

\section{A. Spherical Symmetry}

We begin by solving for spherically symmetric initialdata sets that contain one black hole. In spherical symmetry, the assumption of conformal flatness is no restriction, because any spherically symmetric metric can be made conformally flat through an appropriate radial coordinate transformation. For example, a hypersurface through the Schwarzschild spacetime of constant KerrSchild coordinate time has the induced metric

$$
d s^{2}=\left(1+\frac{2 M}{R}\right) d R^{2}+R^{2} d \Omega^{2},
$$

where $R$ denotes the areal radius. Here, the coordinate transformation [1]

$$
r=\frac{R}{4}\left(1+\sqrt{1+\frac{2 M}{R}}\right)^{2} e^{2-2 \sqrt{1+2 M / R}},
$$

brings the induced metric into conformally flat form,

$$
d s^{2}=\psi_{\mathrm{KS}}^{4}\left(r^{2} d r^{2}+r^{2} d \Omega^{2}\right),
$$

with $\psi_{\mathrm{KS}}=\sqrt{R / r}$.

In order to support our claim that $K$ and the lapse boundary condition merely represent a coordinate choice, we solve the quasiequilibrium equations for several, essentially arbitrary, choices for these quantities. For the mean curvature, we choose

$$
\begin{aligned}
& K=0, \\
& K=\frac{2 M}{r^{2}}, \\
& K=K_{\mathrm{KS}} \equiv \frac{2 M}{R^{2}}\left(1+\frac{2 M}{R}\right)^{-3 / 2}\left(1+\frac{3 M}{R}\right),
\end{aligned}
$$

where in the last case, $R$ is given implicitly by Eq. (56). Equation (58c) represents the mean curvature for a KerrSchild slice of the Schwarzschild spacetime with mass $M$.

For the lapse boundary condition at the excised spheres, we use

$$
\begin{aligned}
& \left.\frac{d \alpha \psi}{d r}\right|_{S}=0, \\
& \left.\frac{d \alpha \psi}{d r}\right|_{S}=\left.\frac{\alpha \psi}{2 r}\right|_{S} \\
& \left.\alpha \psi\right|_{S}=\frac{1}{2}, \\
& \left.\alpha \psi\right|_{S}=\left.\frac{1}{\sqrt{2}} \psi_{\mathrm{KS}}\right|_{S} .
\end{aligned}
$$

The last condition, Eq. (59d), is correct for the KerrSchild slice.

We now compute 12 initial-data sets, combining any of the choices for $K$ with any of the lapse boundary conditions. In order to fully recover the Kerr-Schild slice for the choices (58c) and (59d), we set the radius of the 
excised sphere to

$$
r_{\mathrm{exc}}=\left.r\right|_{R=2 M}=\frac{1}{2}(1+\sqrt{2})^{2} e^{2-2 \sqrt{2}} M .
$$

For each of the initial-data sets, we compute the residual in the Hamiltonian and momentum constraints, Eqs. (5) and (6). We compute Arnowitt-Deser-Misner (ADM) quantities of the initial-data sets by the standard integrals at infinity in Cartesian coordinates,

$$
\begin{gathered}
E_{\mathrm{ADM}}=\frac{1}{16 \pi} \int_{\infty}\left(\gamma_{i j, j}-\gamma_{j j, i}\right) d^{2} S_{i}, \\
J_{(\xi)}=\frac{1}{8 \pi} \int_{\infty}\left(K^{i j}-\gamma^{i j} K\right) \xi_{j} d^{2} S_{i} .
\end{gathered}
$$

For the $x$-component of the linear ADM momentum, $\xi=$ $\hat{e}_{x}$ in Eq. (62). The choice $\xi=x \hat{e}_{y}-y \hat{e}_{x}$ yields the $z$-component of the ADM-like angular momentum as defined by York [33]. We also compute the irreducible mass

$$
M_{\mathrm{irr}}=\sqrt{\frac{A_{A H}}{16 \pi}}
$$

where we have approximated the area of the (unknown) event horizon by the area $A_{A H}$ of the apparent horizon, and the Komar mass,

$$
M_{\mathrm{K}}=\frac{1}{4 \pi} \int_{r=\infty} \gamma^{i j}\left(\bar{\nabla}_{i} \alpha-\beta^{k} K_{i k}\right) d^{2} S_{j} .
$$

Finally, we evaluate the time derivative of $K_{i j}$ by Eq. (4), evaluate the residual $\alpha_{\mathrm{BC}}$ of the quasiequilibrium lapse condition, Eq. (51), and compute

$$
\partial_{t} \ln \psi=-\frac{1}{6}\left(\alpha K-\bar{\nabla}_{i} \beta^{i}\right),
$$

which follows from the trace of Eq. (3).

Figure 1 presents a convergence plot for one of the 12 cases, Eqs. (58c) and (59d). This case recovers the usual Kerr-Schild slice with mass $M$. The residual of the Hamiltonian and momentum constraints decrease exponentially with resolution, and the three different masses $M_{\mathrm{irr}}, E_{\mathrm{ADM}}$, and $M_{\mathrm{K}}$, all converge to the expected result, $M$. Furthermore, the time derivatives exponentially converge to zero, and ADM linear and angular momenta converge to zero, too. The vanishing time derivatives indicate that the quasiequilibrium method constructs lapse and shift along the timelike Killing vector of the Schwarzschild spacetime.

For different choices of $K$ or for the lapse BC, we find that the masses are no longer exactly unity. However, for all choices of $K$ and lapse BC, we find to within truncation error, that the three masses agree,

$$
M_{\text {irr }}=E_{\mathrm{ADM}}=M_{\mathrm{K}},
$$

and that all time derivatives (and the lapse condition

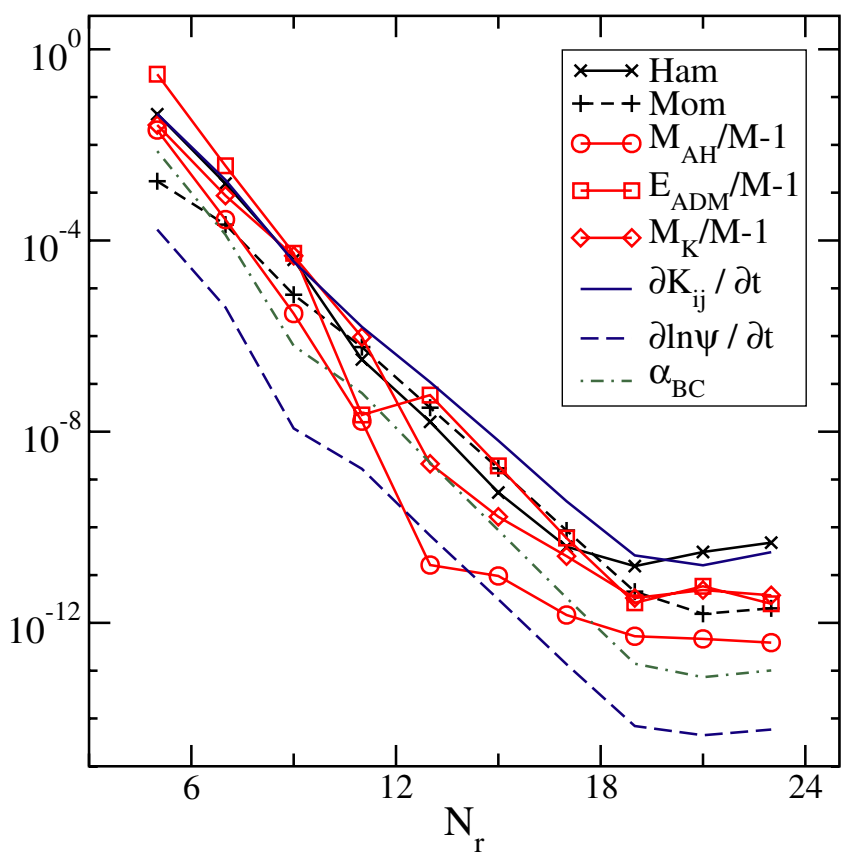

FIG. 1 (color online). Solution of the quasiequilibrium equations recovering the Kerr-Schild slicing of Schwarzschild. Plotted are the maximum values of Hamiltonian and momentum constraints and of time derivatives, as well as the deviation of $M_{\mathrm{irr}}, E_{\mathrm{ADM}}$, and $M_{K}$ from the analytical answer $M . N_{r}$ is the radial number of collocation points in each of the two spherical shells.

Eq. (51)) vanish:

$$
\partial_{t} \psi=\partial_{t} K_{i j}=\alpha_{\mathrm{BC}}=0 .
$$

These findings are summarized in Table I. These runs indicate that any (reasonable) choice for the mean curvature $K$ and the lapse $\mathrm{BC}$ recovers a slice through Schwarzschild with time vector along the timelike Killing vector.

For the maximal slices, $K=0$, the different lapse BCs choose different parameters $C / M^{2}$ in the family of maximal slicings, Eqs. (52a)-(52d). The boundary conditions in Eqs. (59a)-(59d) correspond, respectively, to $C / M^{2}=$ $2 / 3(\sqrt{13}-1), \quad C / M^{2}=4 / 3, \quad C / M^{2} \approx 1.2393, \quad$ and $C / M^{2} \approx 2.4905$. Based on the results of Table I, we conjecture that for any (reasonable) function $K(r)$, there exists a one-parameter family of spherically symmetric slicings, which extend from the horizon to spatial infinity.

In situations with less symmetry like binary black holes, we prefer Neumann or Robin boundary conditions on the lapse (Eqs. (59a) and (59b)), because they allow the lapse on the horizon to respond to tidal deformations. Furthermore, as Table I confirms that the choice of mean curvature plays a marginal role, we will concentrate on the most obvious choice, maximal slicing $K=0$, below. 
TABLE I. Spherically symmetric quasiequilibrium initialdata sets. Given are the irreducible mass, ADM energy, and Komar mass (these three quantities are found to be identical to within truncation error). The last column gives an upper bound on the deviation from zero of Hamiltonian and momentum constraints, time derivatives of $\psi$ and $K_{i j}$, as well as the lapse condition $\alpha_{\mathrm{BC}}$.

\begin{tabular}{|c|c|c|c|}
\hline$K$ & Lapse BC & $M_{\mathrm{irr}}, E_{\mathrm{ADM}}, M_{\mathrm{K}}$ & $\mathcal{H}, \mathcal{M}_{i}, " \partial_{t} "$ \\
\hline \multirow[t]{4}{*}{0} & $(\alpha \psi)^{\prime}=0$ & 1.48079275 & $<10^{-10}$ \\
\hline & $(\alpha \psi)^{\prime}=\alpha \psi /(2 r)$ & 1.61967937 & $<10^{-10}$ \\
\hline & $\alpha \psi=1 / 2$ & 1.65726413 & $<10^{-10}$ \\
\hline & $\alpha \psi=(\alpha \psi)_{\mathrm{KS}}$ & 1.28974831 & $<10^{-10}$ \\
\hline \multirow[t]{4}{*}{$2 M / r^{2}$} & $(\alpha \psi)^{\prime}=0$ & 0.68281 & $<10^{-9}$ \\
\hline & $(\alpha \psi)^{\prime}=\alpha \psi /(2 r)$ & 0.73571 & $<10^{-9}$ \\
\hline & $\alpha \psi=1 / 2$ & 0.99176 & $<10^{-8}$ \\
\hline & $\alpha \psi=(\alpha \psi)_{\mathrm{KS}}$ & 0.77233 & $<10^{-9}$ \\
\hline \multirow[t]{4}{*}{$K_{\mathrm{KS}}$} & $(\alpha \psi)^{\prime}=0$ & 0.9942475 & $<10^{-9}$ \\
\hline & $(\alpha \psi)^{\prime}=\alpha \psi /(2 r)$ & 1.091637 & $<10^{-9}$ \\
\hline & $\alpha \psi=1 / 2$ & 1.295099 & $<10^{-9}$ \\
\hline & $\alpha \psi=(\alpha \psi)_{\mathrm{KS}}$ & 1.0000000 & $<10^{-10}$ \\
\hline
\end{tabular}

In summary, the quasiequilibrium method is singularly successful for spherically symmetric spacetimes: There exists a natural choice for $\tilde{\gamma}_{i j}$ (the flat metric) that, together with any (reasonable) choices for $K$ and the lapse boundary condition, yields a slice though Schwarzschild and the timelike Killing vector that results in a completely time-independent evolution.

\section{B. Single Rotating Black Holes}

In Sec. III we explained that the tangential component of the shift vector on the excised surface induces a rotation on the hole, cf. Equation (50). We now test this assertion by constructing initial-data sets for single rotating black holes.

We set the mean curvature and the lapse $\mathrm{BC}$ by Eqs. (58a) and (59a), and we continue to use the conformal flatness approximation, $\tilde{\gamma}_{i j}=f_{i j}$, and choose the excised region to be a coordinate sphere centered on the origin with radius $r_{\mathrm{exc}}=0.8594997$. This value ensures a unit-mass black hole in the limit of no rotation for the choice of $K$ and lapse BC. The shift boundary conditions encode the rotation. At infinity, we set $\beta^{i}=0$; on the horizon, Eq. (50) implies

$$
\beta_{\|}^{i}=\Omega_{r}^{j} x^{k} \epsilon^{i j k},
$$

where $x^{k}$ is the Cartesian coordinate separation of points on $S$ to the center of the excised sphere. We choose $\Omega_{r}^{i}$ parallel to the $\mathrm{z}$-axis, and solve the initial value equations for different magnitudes of $\Omega_{r}$. For each solution, we compute the diagnostics mentioned in Sec. IVA. Figure 2 presents the ADM energy, irreducible mass, and angular momentum of the obtained data sets. We

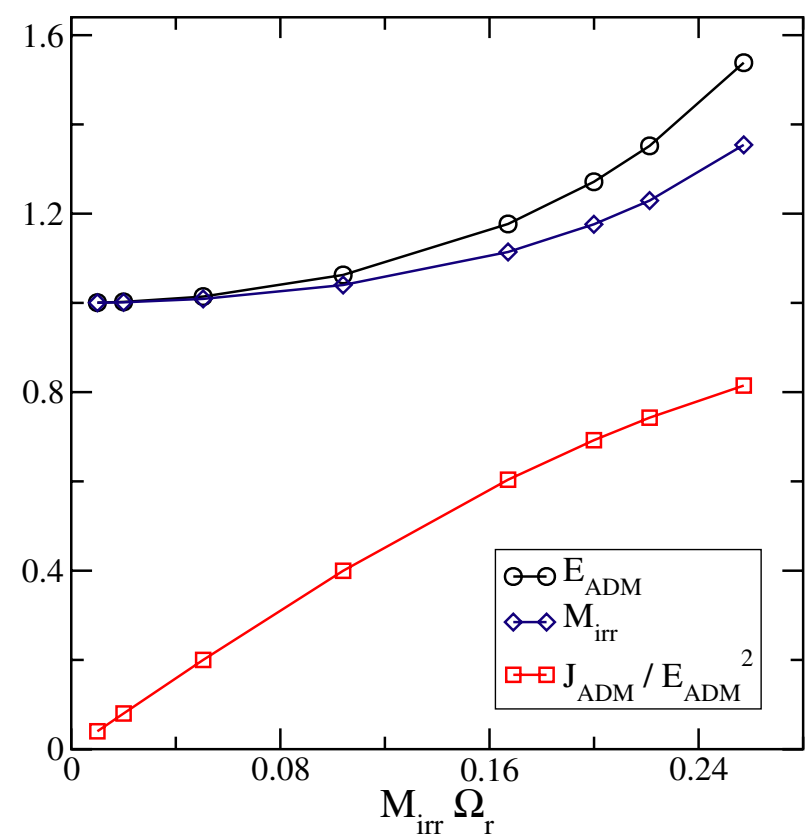

FIG. 2 (color online). Spinning single black hole initial-data sets.

see that, for small $\Omega_{r}$, the angular momentum increases linearly with $\Omega_{r}$, as expected.

As discussed early in Sec. IV, our assumption of conformal flatness will necessarily introduce some errors when solving for a rotating black hole, because the Kerr metric does not admit conformally flat slices[26,27]. Very interesting are, therefore, measures of the deviation of the quasiequilibrium initial-data sets to a slice through the exact Kerr spacetime.

One such quantity is the maximum amount of energy that can potentially be radiated to infinity,

$$
E_{\mathrm{rad}}=\sqrt{E_{\mathrm{ADM}}^{2}-P_{\mathrm{ADM}}^{2}}-\sqrt{M_{\mathrm{irr}}^{2}+\frac{J_{\mathrm{ADM}}^{2}}{4 M_{\mathrm{irr}}^{2}}} .
$$

For a stationary spacetime, $E_{\mathrm{rad}}=0$. Another interesting question is how closely $\Omega_{r}$ of Eq. (68) corresponds to the angular frequency of the horizon. For a Kerr black hole with angular momentum $J_{\mathrm{ADM}}$ and total mass $E_{\mathrm{ADM}}$, the angular frequency of the horizon is given by [34]

$$
\Omega_{H}=\frac{J_{\mathrm{ADM}} / E_{\mathrm{ADM}}^{3}}{2+2 \sqrt{1-\left(J_{\mathrm{ADM}} / E_{\mathrm{ADM}}^{2}\right)^{2}}},
$$

so that

$$
\Delta \Omega \equiv E_{\mathrm{ADM}}\left(\Omega_{r}-\Omega_{H}\right)
$$

measures the deviation of $\Omega_{r}$ from the angular frequency of the horizon. Figure 3 presents these quantities. The maximum radiation content, $E_{\mathrm{rad}}$, is proportional to $\left(M_{\text {irr }} \Omega_{r}\right)^{4}$. For the binary black-hole data sets we con- 


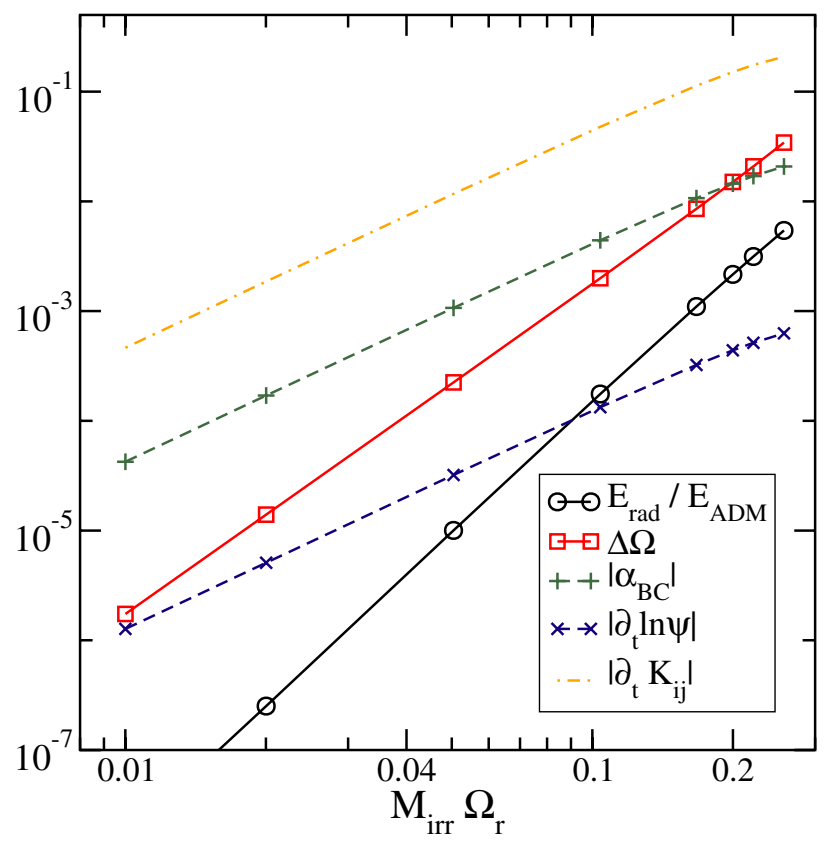

FIG. 3 (color online). Single rotating black hole initial-data sets: Deviations from the exact Kerr metric.

struct below in Sec. V, the relevant angular frequency is the orbital angular frequency. We find below, that at the innermost stable circular orbit, $M_{\text {irr }} \Omega_{0} \sim 0.11$. From Fig. 3, we find for this angular frequency, $E_{\mathrm{rad}} / E_{\mathrm{ADM}} \approx$ $2 \cdot 10^{-4}$. This indicates that, when conformal flatness is assumed, we should not expect the fractional error induced in $E_{\mathrm{ADM}}$ for systems with nonvanishing angular momentum to be larger than $\sim 10^{-3}$. Furthermore, at $M_{\text {irr }} \Omega_{r}=0.11, \Delta \Omega \approx 0.0025$. From this we expect that the rotational state of each black hole, within the binary black-hole configurations below, should deviate by at most $1 \%$ or $2 \%$ from the intended values for corotating or irrotational holes. In Fig. 3 we also plot some time derivatives assuming the initial data are evolved with the constructed gauge $\alpha, \beta^{i}$. These time derivatives are proportional to $\left(M_{\mathrm{irr}} \Omega_{r}\right)^{2}$. However, their interpretation is more difficult, due to their gauge dependence and the difficulty of finding a meaningful normalization.

\section{Single Boosted Black Holes}

A boosted single black hole in a comoving coordinate system appears time-independent. A well-known example is the boosted Kerr-Schild form of a Kerr black hole. In such comoving coordinates, the shift does not vanish at infinity, but approaches the boost velocity of the black hole,

$$
\left.\beta^{i}\right|_{r \rightarrow \infty}=v^{i}
$$

We apply now the quasiequilibrium formalism to construct boosted black holes in comoving coordinates by using Eq. (72) as the boundary condition on the shift at the outer boundary. At the excised sphere, we set $\left.\beta_{\|}^{i}\right|_{S}=$ 0 . Furthermore, we assume again conformal flatness, use Eqs. (58a) and (59a) to fix the mean curvature and the lapse BC, and excise a coordinate sphere with radius $r_{\text {exc }}$. The remaining free parameter is the magnitude of the boost velocity, $v$. Figure 4 presents the ADM energy, irreducible mass, and $P_{\mathrm{ADM}} / E_{\mathrm{ADM}}$ as a function of the boost velocity. $P_{\mathrm{ADM}} / E_{\mathrm{ADM}}$ is linear in $v$ for small $v$, as it should be. As $v$ approaches unity, $E_{\mathrm{ADM}}$ strongly increases. Figure 5 presents measures of how faithfully these initial-data sets represent a boosted stationary black hole. The maximum radiation content $E_{\mathrm{rad}}$ grows as $v^{4}$. At the ISCO we can estimate that $v \sim 0.4$ where we find $E_{\mathrm{rad}} \approx 10^{-3} E_{\mathrm{ADM}}$. In order to measure how well the special relativistic relation $v=P / E$ is satisfied, we define

$$
\Delta v \equiv v-\frac{P_{\mathrm{ADM}}}{E_{\mathrm{ADM}}}
$$

we find that $\Delta v \propto v^{3}$ for small $v$, with $\Delta v \approx 0.01$ for $v=0.4$.

\section{QUASICIRCULAR ORBITS FOR BLACK-HOLE BINARIES}

In the case of a single black hole, if appropriate choices for the freely specifiable data and boundary conditions are made, then an exact equilibrium solution of the initial-data equations can be found. However, for binary black-hole configurations, no such true equilibrium or stationary state exists. This is a much more stringent

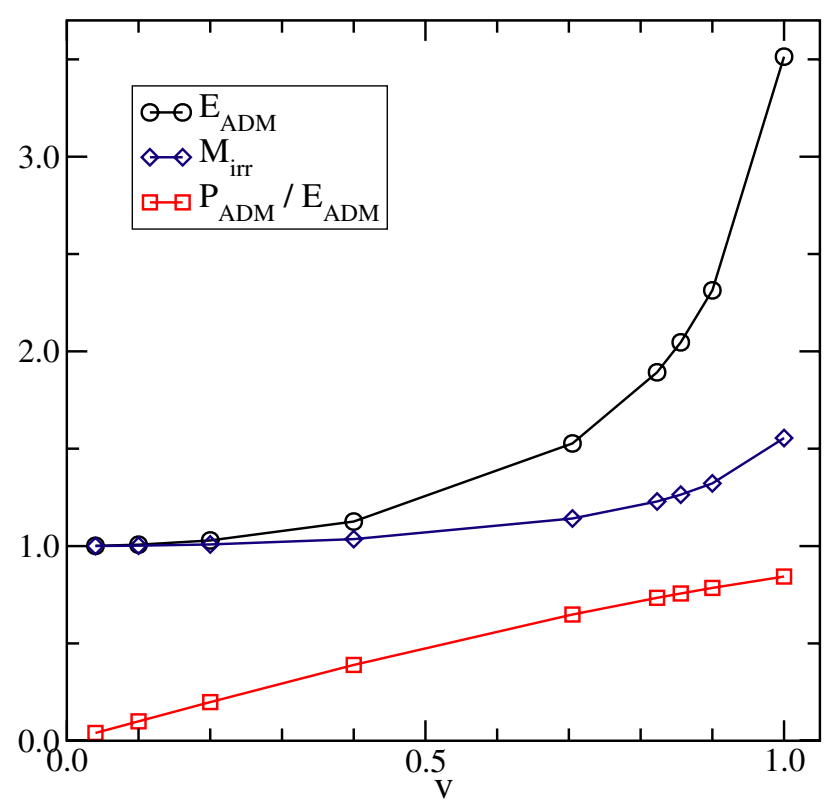

FIG. 4 (color online). Single boosted black hole initial-data sets. 


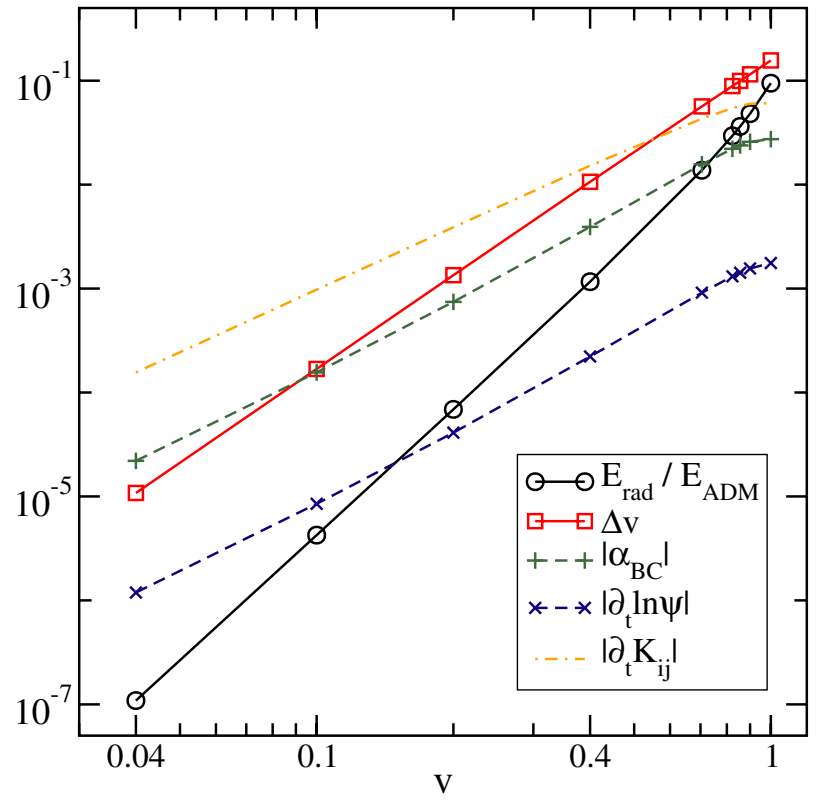

FIG. 5 (color online). Single boosted black hole initial-data sets: Deviations from exact time-independence.

test of the quasiequilibrium boundary conditions. In this section, we will examine the solutions for the case of equal-mass black-hole binaries that are either corotating or irrotational.

We will consider binary configurations over a range of separations. A black hole is represented in the coordinate system by an excised two-surface. The relative coordinate sizes of various excised surfaces parametrize the relative sizes of the resulting physical black holes. The coordinate separations of the holes parametrize their physical separation. These coordinate sizes and separations are measured in the coordinates associated with the chosen conformal metric. In this work, all excised surfaces are the surfaces of coordinate spheres. Furthermore, for the simple cases of corotating and irrotational binaries, equal-mass black holes are obtained by choosing excision surfaces for the two holes that have equal radii.

Before solving the initial-data equations, we must make choices for the freely specifiable data. In all cases, we will make use of the quasiequilibrium assumptions on the free data that $\tilde{u}_{i j}=0$ and $\partial_{t} K=0$. We also continue to use the approximation that the conformal threegeometry is flat (i.e., that $\tilde{\gamma}_{i j}$ is a flat metric). The remaining free data is the trace of the extrinsic curvature, $K$. For this, we will consider two choices: maximal slicing with $K=0$, and a nonmaximal slicing based on EddingtonFinkelstein slicing.

In addition to the freely specifiable data, we must also fix the boundary conditions on the excision surfaces that correspond to the surface of each black hole and at the outer boundary of the computational domain. The outer boundary conditions were discussed at the end of Sec. II. The boundary condition on the shift, Eq. (16b), contains a free parameter $\Omega_{0}$ that determines the orbital angular velocity of the system. The value of this parameter is chosen by demanding that the ADM and Komar masses of the system must be equal $[1,9,18]$. This is a quasiequilibrium condition that is satisfied by a single value of $\Omega_{0}$ and places the binary in a nearly circular orbit.

For the excision boundaries, we will use the apparent horizon condition given by Eq. (48) as a boundary condition of the conformal factor $\psi$, and we will use Eq. (39) to fix the component of the shift that is normal to the excision surface. Boundary conditions on the components of the shift that are tangent to the excision surface depend on our choice for the spins of the black holes. For the case of corotation, we will demand that $\beta_{\|}^{i}=0$. However, the irrotational case requires a somewhat more complicated choice.

The condition of quasiequilibrium requires that we choose the tangential components of the shift so that they have the form given in Eq. (50). For irrotational black holes in a binary, it is reasonable to choose the conformal Killing vector $\xi^{i}$ so that it represents rotation about an axis that is orthogonal to the plane of the orbit. If we let $\Omega_{r}^{i}$ represent an angular velocity vector that is orthogonal to the plane of the orbit, and if we use Cartesian coordinates for our flat conformal metric, then Eq. (50) can be written as

$$
\beta_{\|}^{i}=\Omega_{r}^{j} x_{ \pm}^{k} \epsilon^{i j k}
$$

where $x_{ \pm}^{i} \equiv x^{i}-C_{ \pm}^{i}$ and $C_{ \pm}^{i}$ is the Cartesian coordinate location of the center of either of two excision spheres. Finally, we take the magnitude of $\Omega_{r}^{i}$ to be equal to the orbital angular velocity of the binary system as measured at infinity, $\Omega_{0}$. We note that there is no rigorous proof that these choices lead to an irrotational binary system. However, as argued in Sec. III B, especially the paragraphs leading to Eq. (43), these choices seem reasonable.

Finally, we must choose a boundary condition on the lapse at the excision boundaries. For all choices of the black-hole spins and choices for $K$, we repeat the computations for three different lapse boundary conditions, namely, Eqs. (59a)-(59c).

The conformal thin-sandwich equations are solved with the pseudospectral collocation method described in [32]. The computational domain consists of one inner spherical shell around each excised sphere, which overlap 43 rectangular blocks, which in turn overlap an outer spherical shell extending to $r_{\text {out }}=10^{9}$. Figure 6 shows convergence of this solver with spatial resolution for one typical configuration (separation $d=9, K=0$, corotating black holes). The calculations below are performed at a resolution comparable to $N=60$, so that the discretization errors in $E_{\mathrm{ADM}}$ and $M_{\mathrm{K}}$ should be about $10^{-6}$. 


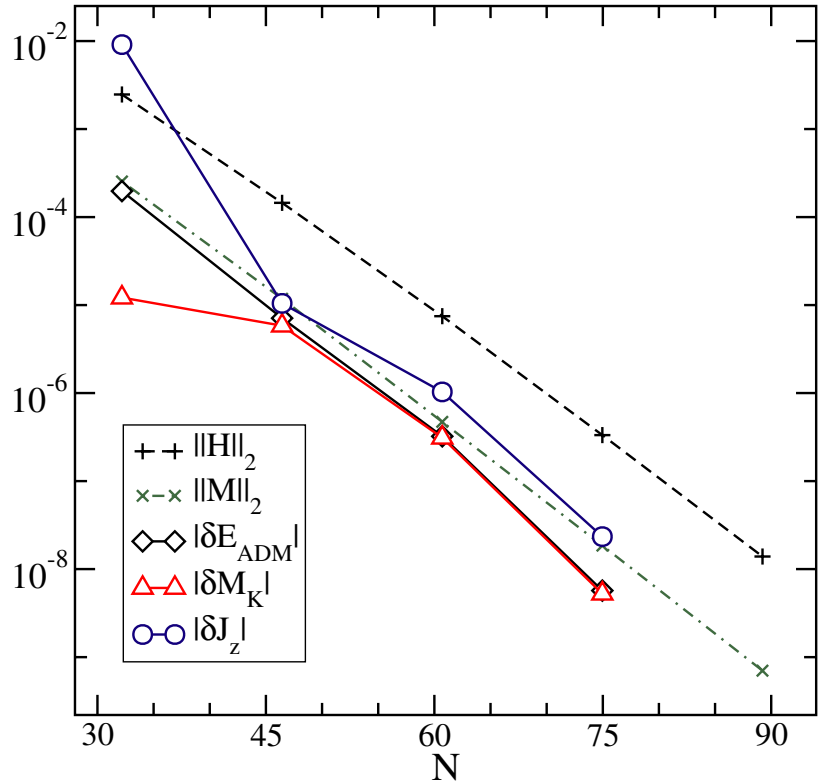

FIG. 6 (color online). Convergence of the elliptic solver for the binary black-hole configurations. Plotted are the constraint violations in Hamiltonian and momentum constraint, and differences to the highest resolution solved. $N$ is the cuberoot of the total number of grid-points.

\section{A. Maximal Slicing}

\section{Corotating binary systems}

We now compute initial-data sets corresponding to a binary black-hole system in a quasicircular orbit for many different separations. Figure 7 shows the binding energy $E_{b}$ of the binary system as a function of the total angular momentum of the system for all the lapse boundary conditions. The binding energy is defined as $E_{b} \equiv$ $E_{\mathrm{ADM}}-m$ where $E_{\mathrm{ADM}}$ is the total (ADM) energy of the system and $m=m_{1}+m_{2}$ is the total mass of the system. For the quasiequilibrium numerical results described in this paper, we take $m_{1 \mid 2} \equiv \sqrt{\left(A_{1 \mid 2} / 16 \pi\right)}$ as the irreducible mass of each individual black hole and $A_{1 \mid 2}$ are the areas of the apparent horizon of each hole. The reduced mass is defined as $\mu \equiv\left(m_{1} m_{2} / m\right)$.

We note that the choice of the lapse boundary condition has very little effect on the solutions. This is consistent with our assertion that the choice of the lapse boundary condition is part of the initial temporal gauge choice. The inset in Fig. 7 shows a magnified view of the region where the black holes are closest to each other. Even in this region, the result of the different lapse boundary conditions are nearly indistinguishable. Because of this, subsequent plots displaying corotating maximal slicing results will only display one of these sequences.

Figure 8 shows a comparison of the same data to analogous results obtained by Grandclément et. al. [18] (labeled CO:HKV-GGB in figure legends), and effective

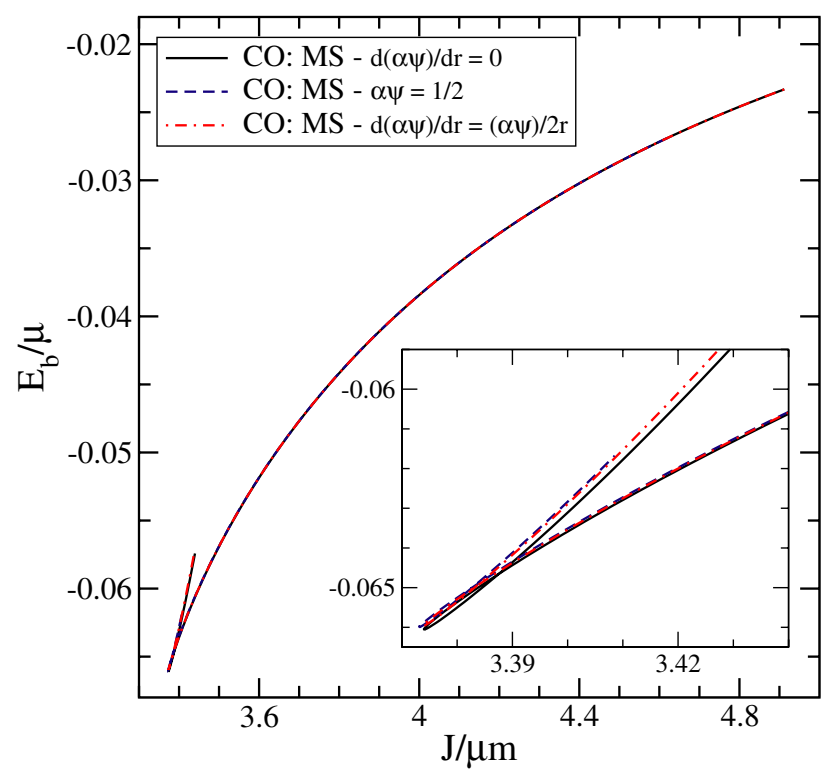

FIG. 7 (color online). Constant $M_{\text {irr }}$ sequence of corotating equal-mass black holes. Maximal slicing is used in these cases, and three different excision boundary conditions for the lapse are used.

one-body post-Newtonian results as reported in Ref. [35]. First, second, and third post-Newtonian (PN) results are displayed (labeled CO:EOB-1PN, CO:EOB-2PN, and CO:EOB-3PN, respectively, in figure legends). The 3PN results correspond to the approach labeled "3PN corot. $\bar{A}\left(u, \hat{a}^{2}\right) "$ in Table I of Ref. [35].

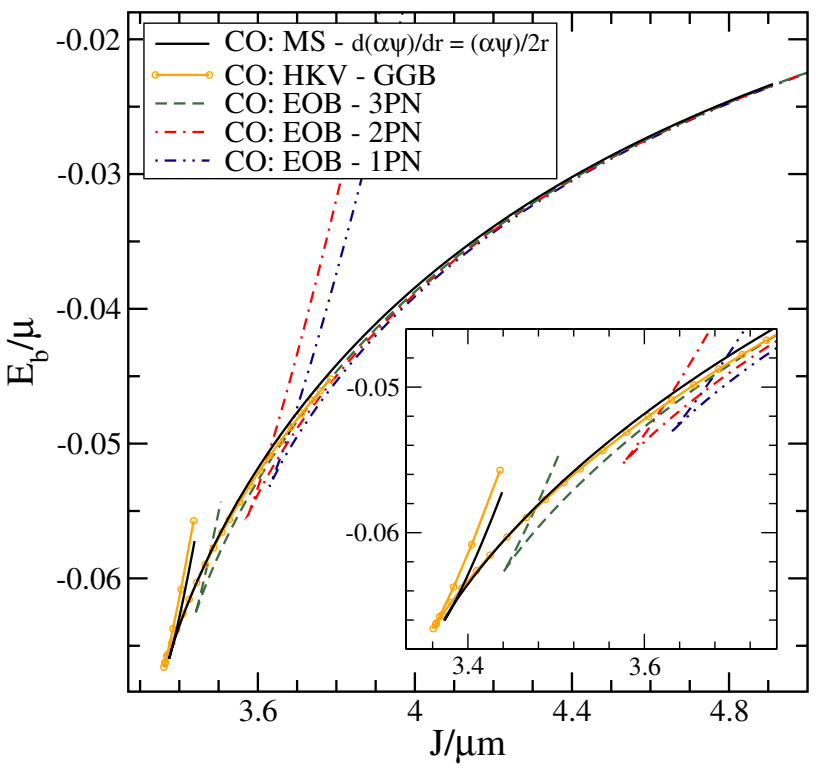

FIG. 8 (color online). Constant $M_{\text {irr }}$ sequence of corotating equal-mass black holes. Comparison of post-Newtonian EOB sequences with numerical maximal slicing results from HKV and this paper. 
There is good agreement between all of the results at large separation. Also, it appears that the PN results are converging toward the quasiequilibrium numerical results, even when the black holes are quite close to each other as seen in the figure's inset. We also see that the numerical results obtained by Grandclément et. al. [18] (hereafter GGB) differ only slightly from the quasiequilibrium results. As discussed in Ref. [1], the numerical solutions obtained by GGB must violate the constraints. The agreement seen in Fig. 8 lends support to the belief that the violation of the constraints is, in some sense, small and has a small impact on the physical content of the data.

Another method for comparing data for the circular orbits of compact binaries is to examine the location of the innermost stable circular orbit (ISCO). The ISCO is not a well-defined concept in general. However, in situations where the dissipative effects of radiation reaction have been eliminated, an ISCO becomes more meaningful. The ISCO is defined in terms of a minimum of some appropriate energy. For corotating binary systems, true stationary configurations can exist (although they contain an infinite amount of energy in the form of gravitational radiation; see Ref. [36]). In this case, the minimum of the total energy can be rigorously associated with the onset of a secular instability [37,38]. In the absence of true stationary configurations, this "turning-point" method is still used to define the ISCO.

In order to locate a turning point, one must have a sequence of binary configurations spanning a range of separations. How this sequence is constructed is not uniquely defined. The ambiguity arises because of the lack of a fixed fundamental length scale in the problem. In the first work to construct sequences of black-hole binary initial-data sets representing circular orbits [39], the total mass of the black hole (defined in terms of the Christodoulou mass formula [40]) was used to normalize the sequences.

GGB suggest another approach based on the thermodynamic identity [36]

$$
\mathrm{d} E_{\mathrm{ADM}}=\Omega_{0} \mathrm{~d} J_{\mathrm{ADM}} .
$$

This identity should be satisfied by a true stationary sequence of corotating black holes. Let $s$ denote some parameter along a sequence of initial-data sets, and let $e(s), j(s)$, and $\omega(s)$ denote the numerical values for dimensionless versions of the total energy, total angular momentum, and orbital angular velocity at location $s$ along the sequence. We are free to define a fundamental length scale $\chi(s)$ along the sequence in any way we like, so long as we define the dimensionful total energy $E_{\mathrm{ADM}}(s)$, total angular momentum $J_{\mathrm{ADM}}(s)$, and orbital angular velocity $\Omega_{0}(s)$ consistently via

$$
E_{\mathrm{ADM}}(s) \equiv \chi(s) e(s)
$$

$$
\begin{aligned}
J_{\mathrm{ADM}}(s) & \equiv \chi^{2}(s) j(s), \\
\Omega_{0}(s) & \equiv \chi^{-1}(s) \omega(s) .
\end{aligned}
$$

Enforcing the identity (75) is sufficient to determine the change in $\chi(s)$ between two points on the sequence. If we integrate along the sequence from a point $s_{1}$ to another point $s_{2}$, then we find that

$$
\chi\left(s_{2}\right)=\chi\left(s_{1}\right) \exp \left\{-\int_{s_{1}}^{s_{2}} \frac{e^{\prime}(s)-\omega(s) j^{\prime}(s)}{e(s)-\omega(s) j(s)} d s\right\},
$$

where a prime denotes differentiation along the sequence.

If the sequence is normalized via Eq. (75), then the irreducible mass of one black hole, $M_{\text {irr }}=\frac{1}{2} m$, is not necessarily constant along the sequence. The top half of Fig. 9 shows $M_{\text {irr }}$ for a corotating quasiequilibrium equalmass binary as a function of the orbital angular velocity. The length scale has been normalized so that $M_{\text {irr }}=1 / 2$ at infinite separation. We confirm the finding of GGB that $M_{\text {irr }}$ is nearly constant along the sequence. While there is a clear increase in the mass as the separation decreases, this increase is small and appears to be of roughly the same order of magnitude as the differences due to using different lapse boundary conditions. As we will see later, this behavior is not mirrored in the irrotational data.

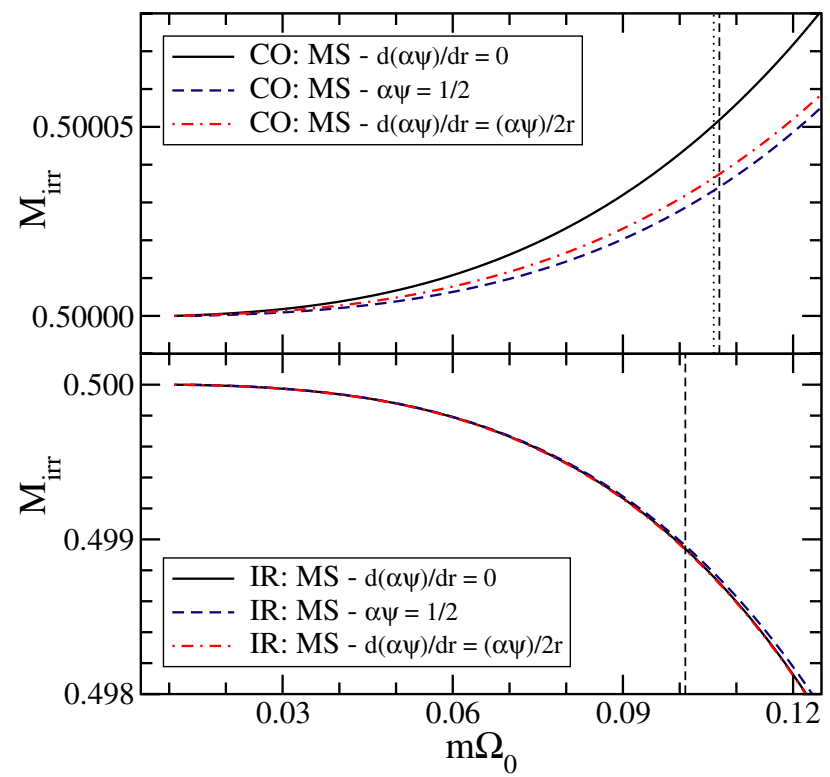

FIG. 9 (color online). Plot of the irreducible mass of one black hole when the sequence of solutions is normalized to maintain $\mathrm{d} E=\Omega \mathrm{d} J$. Three different choices for the lapse boundary condition are shown for both corotating (upper plot) and irrotational (lower plot) black holes in an equalmass binary. The vertical dashed line shows the approximate location of the ISCO defined by a minimum in $E_{b}$. The vertical dotted line shows the approximate location of the ISCO defined by a minimum in $E_{\mathrm{ADM}}$. In the irrotational case, this latter ISCO line is off the plot to the right. 
Yet another approach for normalizing the sequence is to demand that the individual irreducible masses associated with the apparent horizons remain constant. This is a particularly convenient normalization for numerical work since it relies on a well-defined and easily measured geometric quantity. Each of these choices for normalizing an initial-data sequence can affect the location of the ISCO, so it is important to use a consistent definition when comparing data.

It is also important to clearly define which energy is being extremised when using a turning-point method to locate the ISCO. We can consider using the minimum in either the ADM energy $E_{\mathrm{ADM}}$ or the binding energy $E_{b}$ along any sequence to define the ISCO. From the definition of the binding energy as $E_{b} \equiv E_{\mathrm{ADM}}-m$, we see that the minima will not necessarily agree if $m$ varies along the sequence.

For the PN sequences, the ISCO is defined as the minimum in the binding energy $E_{b}$ along sequences where the irreducible masses of the black holes remain fixed. For the PN sequences, Eq. (75) is identically satisfied as well, so this is equivalent to finding the minimum in $E_{\mathrm{ADM}}$. This is not true for the quasiequilibrium numerical data.

Table II displays the dimensionless orbital angular velocity, binding energy, and total angular momentum of the ISCO for corotating equal-mass black holes on a maximal slice. For the quasiequilibrium data defined in this paper, two definitions of the ISCO are listed. One uses the minimum in $E_{\mathrm{ADM}}$ along sequences where condition (75) is satisfied to define the ISCO. The alternative

TABLE II. Parameters of the ISCO configuration for corotating equal-mass black holes computed with the maximal slicing condition. Results are given for three different choices of the lapse boundary condition and two choices for the definition of the location of the ISCO. For comparison, the lower part of the table lists results of Refs. [18,35,41]; "PN standard" [41] represents a post-Newtonian expansion in the standard form without use of the EOB technique.

\begin{tabular}{lcccc}
\hline \hline Lapse BC & ISCO min. & $m \Omega_{0}$ & $E_{b} / m$ & $J / m^{2}$ \\
\hline$\frac{\mathrm{d}(\alpha \psi)}{(\mathrm{d} r)}=0$ & $\mathrm{ADM}$ & 0.105 & -0.0165 & 0.844 \\
& $E_{b}$ & 0.107 & -0.0165 & 0.844 \\
$\alpha \psi=\frac{1}{2}$ & $\mathrm{ADM}$ & 0.106 & -0.0165 & 0.843 \\
& $E_{b}$ & 0.107 & -0.0165 & 0.843 \\
$\mathrm{~d}(\alpha \psi)$ & $(\mathrm{d} r)$ \\
& $\mathrm{ADM}$ & 0.106 & -0.0165 & 0.843 \\
& $E_{b} r$ & 0.107 & -0.0165 & 0.843 \\
& & 0.103 & -0.017 & 0.839 \\
HKV-GGB & & 0.0667 & -0.0133 & 0.907 \\
1PN EOB & & 0.0715 & -0.0138 & 0.893 \\
2PN EOB & & 0.0979 & -0.0157 & 0.860 \\
3PN EOB & & 0.5224 & -0.0405 & 0.621 \\
1PN standard & & 0.0809 & -0.0145 & 0.882 \\
2PN standard & & 0.0915 & -0.0153 & 0.867 \\
3PN standard & & & &
\end{tabular}

method uses a minimum in $E_{b}$ along sequences where $M_{\text {irr }}$ is held fixed. However, we note that the minima in $E_{b}$ along sequences that satisfy (75) are numerically indistinguishable from the latter. The ISCO for the GGB data (listed as HKV-GGB in the table) is defined as the minima in $E_{b}$ along a sequence where $M_{\text {irr }}$ remains fixed. Recall that the definition of the ISCO for the PN data is consistent with either definition used for the quasiequilibrium data. In addition to the effective one-body (EOB) PN data displayed previously in Fig. 8, we also include "standard" PN results for the ISCO as reported in Ref. [41].

For corotating quasiequilibrium data, there is very little difference in the results for the two definitions of the ISCO. As we will see later, this is not true for the irrotational configurations (see Table III). In that case, it is clear that only the definition in terms of $E_{b}$ is consistent with the PN data.

Figure 10 plots binding energy versus orbital angular velocity for the ISCO obtained for all three lapse boundary conditions for the corotating quasiequilibrium $(\mathrm{QE})$ data, as well as the corotating results from GGB and PN results. All the numerical results are computed on a maximal slice. We see that the results for the different lapse boundary conditions are essentially indistinguishable. We also see that the PN results converge roughly toward the numerical quasiequilibrium results. While we would not expect the quasiequilibrium numerical results to agree with any of the individual PN results, we might expect the GGB result to agree within numerical error. All of our numerical results using different lapse boundary conditions are essentially indistinguishable. Furthermore, if we use a lapse boundary condition that approaches a Dirichlet value of zero on the excision surface, the resulting set of boundary conditions is

TABLE III. Parameters of the ISCO configuration for irrotational equal-mass black holes computed with the maximal slicing condition. Results are given for three different choices of the lapse boundary condition and two choices for the definition of the location of the ISCO. Layout as in Table II.

\begin{tabular}{|c|c|c|c|c|}
\hline Lapse BC & ISCO min. & $m \Omega_{0}$ & $E_{b} / m$ & $J / m^{2}$ \\
\hline \multirow{2}{*}{$\frac{\mathrm{d}(\alpha \psi)}{(\mathrm{d} r)}=0$} & ADM & 0.144 & -0.0146 & 0.761 \\
\hline & $E_{b}$ & 0.101 & -0.0181 & 0.767 \\
\hline \multirow[t]{2}{*}{$\alpha \psi=\frac{1}{2}$} & ADM & 0.148 & -0.0145 & 0.760 \\
\hline & $E_{b}$ & 0.103 & -0.0181 & 0.765 \\
\hline \multirow{2}{*}{$\frac{\mathrm{d}(\alpha \psi)}{(\mathrm{d} r)}=\frac{\alpha \psi}{2 r}$} & $\mathrm{ADM}$ & 0.145 & -0.0146 & 0.761 \\
\hline & $E_{b}$ & 0.101 & -0.0181 & 0.766 \\
\hline \multicolumn{2}{|l|}{ Conf. Imag. } & 0.166 & -0.0225 & 0.744 \\
\hline \multicolumn{2}{|l|}{$1 \mathrm{PN}$ EOB } & 0.0692 & -0.0144 & 0.866 \\
\hline \multicolumn{2}{|l|}{ 2PN EOB } & 0.0732 & -0.0150 & 0.852 \\
\hline \multicolumn{2}{|l|}{ 3PN EOB } & 0.0882 & -0.0167 & 0.820 \\
\hline \multicolumn{2}{|l|}{ 1PN standard } & 0.5224 & -0.0405 & 0.621 \\
\hline \multicolumn{2}{|l|}{ 2PN standard } & 0.1371 & -0.0199 & 0.779 \\
\hline \multicolumn{2}{|l|}{ 3PN standard } & 0.1287 & -0.0193 & 0.786 \\
\hline
\end{tabular}




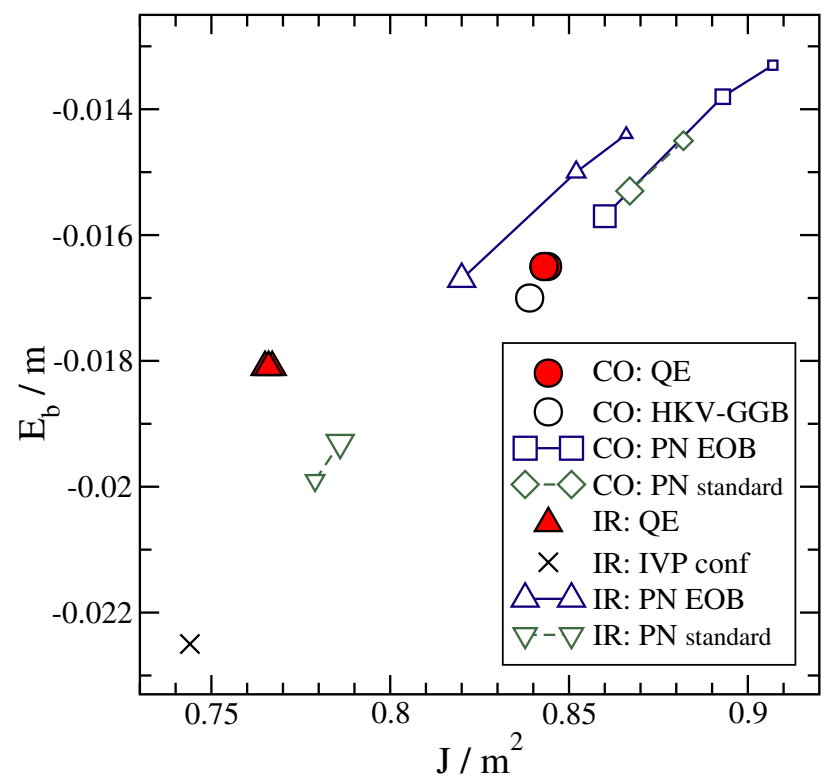

FIG. 10 (color online). ISCO configuration for three different choices of the lapse boundary condition for equal-mass corotating (CO: QE) and irrotational (IR: EQ) black holes computed with the maximal slicing condition. For comparison, results of Refs. [18,35,39,41] are included. For postNewtonian calculations the size of the symbol indicates the order, the largest symbol being 3PN. "PN standard" [41] represents a $\mathrm{PN}$-expansion in the standard form without use of the EOB-technique (only $2 \mathrm{PN}$ and $3 \mathrm{PN}$ are plotted).

equivalent to those used by GGB. The difference seen between the GGB ISCO and our results may well be due to the regularization procedure introduced by GGB.

There are three rigorously defined gauge-invariant global quantities associated with a black-hole binary system: the ADM energy $E_{\mathrm{ADM}}$, the total angular momentum $J$, and the orbital angular velocity as seen at infinity $\Omega_{0}$. Figs. 8 and 10 plot the binding energy $E_{b}$ (directly related to the ADM energy) as a function of $J$. Figs. 11 and 12 plot $E_{b}$ as a function of $\Omega_{0}$ for the same set of sequences and for the ISCO. And, Figs. 13 and 14 plot $J$ as a function of $\Omega_{0}$ for the same set of sequences and for the ISCO.

\section{Irrotational binary systems}

For the case of irrotational black holes, Fig. 15 shows the binding energy $E_{b}$ of the binary system as a function of $J$ for all the lapse boundary conditions. As with the corotating black holes, we again see that the choice of the lapse boundary condition has very little effect on the sequence. In the inset to the figure we see that the effect is largest at small separations and that the differences due to varying the lapse boundary condition are somewhat larger than in the corotating case.

Perhaps the most striking difference between the corotating and irrotational sequences is that the extrema in $E_{b}$

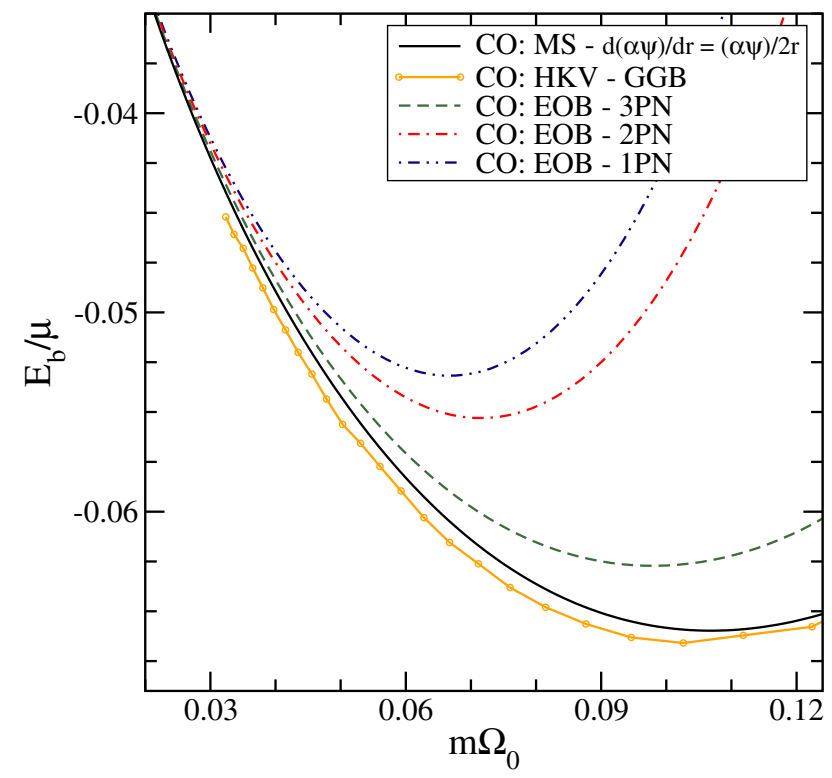

FIG. 11 (color online). Constant $M_{\text {irr }}$ sequence of corotating equal-mass black holes. Comparison of post-Newtonian EOB sequences with numerical maximal slicing results from $\mathrm{HKV}$ and this paper.

versus $J$ are much less "sharp" in the irrotational sequences. If we consider sequences, either corotating or irrotational, that are normalized so that $\mathrm{d} E_{\mathrm{ADM}}=\Omega_{0} \mathrm{~d} J$ is satisfied, then the extremum in $E_{\mathrm{ADM}}$ will necessarily coincide with the extremum in $J$ leading to a very sharp cusp in a plot of these quantities. However, because $M_{\text {irr }}$ is

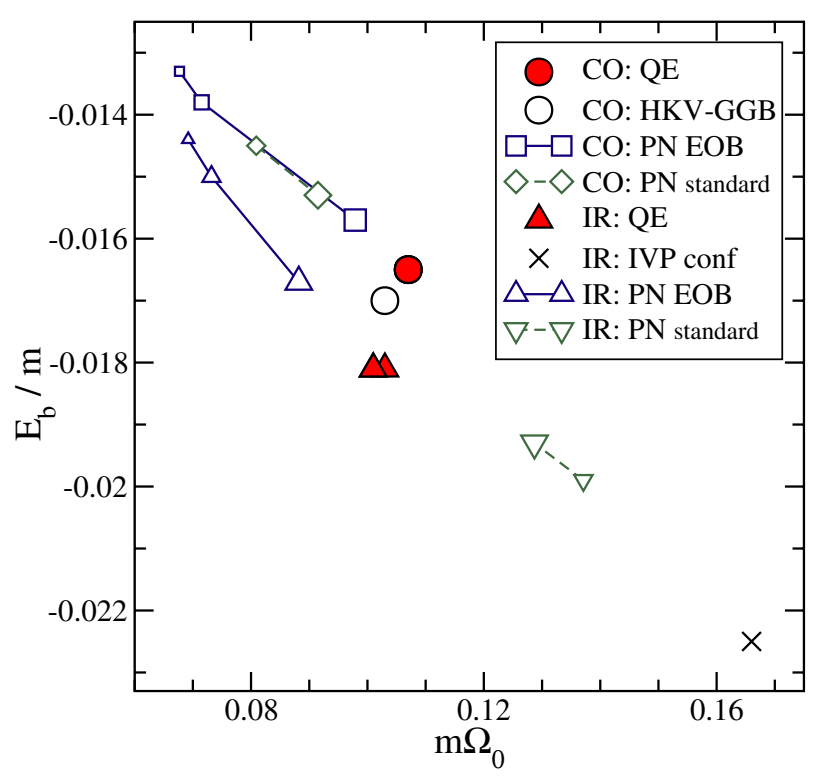

FIG. 12 (color online). ISCO configuration for three different choices of the lapse boundary condition for equal-mass corotating and irrotational black holes computed with the maximal slicing condition. Symbols as in Fig. 10. 


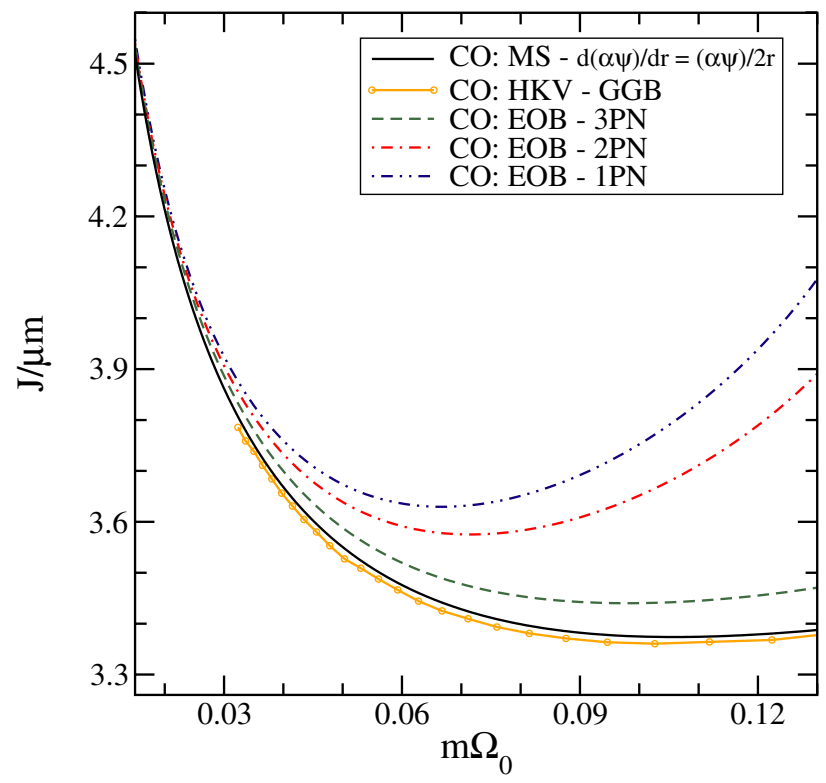

FIG. 13 (color online). Constant $M_{\text {irr }}$ sequence of corotating equal-mass black holes. Comparison of post-Newtonian EOB sequences with numerical maximal slicing results from $\mathrm{HKV}$ and this paper.

not necessarily constant along a sequence with this normalization, the extremum in $E_{b}$ will not necessarily coincide with that of $J$. Thus, we should certainly not expect to see a sharp cusp in either Fig. 7 or Fig. 15.

Another difference between the corotating and irrotational sequences can be seen in Fig. 9. Here we see that, if

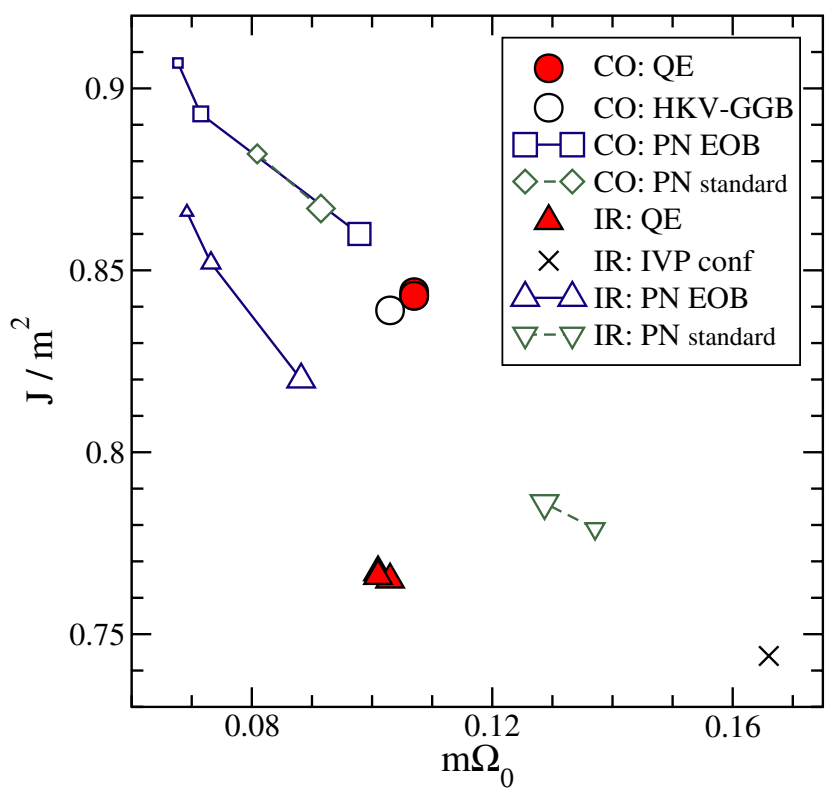

FIG. 14 (color online). ISCO configuration for three different choices of the lapse boundary condition for equal-mass corotating and irrotational black holes computed with the maximal slicing condition. Symbols as in Fig. 10. we demand that the thermodynamic identity (75) be satisfied along the sequence, then the variation in $M_{\mathrm{irr}}$ is 20 times larger in the irrotational sequences than in the corotating sequences. For the corotating sequences, the variation in $M_{\text {irr }}$ due to differences in the lapse boundary condition is comparable to the average variation. For the irrotational sequences, the effect of different lapse boundary conditions is clearly negligible. Furthermore, we note that $M_{\text {irr }}$ is decreasing as the binary separation decreases. This behavior is unphysical, as the irreducible mass never decreases; therefore, $M_{\text {irr }}$ should also not decrease during the inspiral of a binary black hole.

Figure 16 shows a comparison of our irrotational data to the effective one-body post-Newtonian results for irrotational holes as reported in Ref. [35]. First, second, and third post-Newtonian results are displayed (labeled IR:EOB-1PN, IR:EOB-2PN, and IR:EOB-3PN, respectively, in figure legends). The $3 \mathrm{PN}$ results correspond to the approach labeled "3PN corot. $\bar{A}(u, 0)$ " in Table I of Ref. [35]. Also plotted in this figure is the first sequence of numerical initial-data solutions for an equal-mass blackhole binary in quasicircular orbit, obtained from inversion-symmetric initial data using an effective potential approach [39] (labeled IR: Conf. Imaging/Eff. Pot. or IVP conf in figure legends).

Again, there is good agreement between all of the results at large separation. Also, it appears that the PN results are converging toward the irrotational quasiequilibrium numerical results, even when the black holes are quite close to each other as seen in the figure's inset. We also see that the early numerical results obtained from the

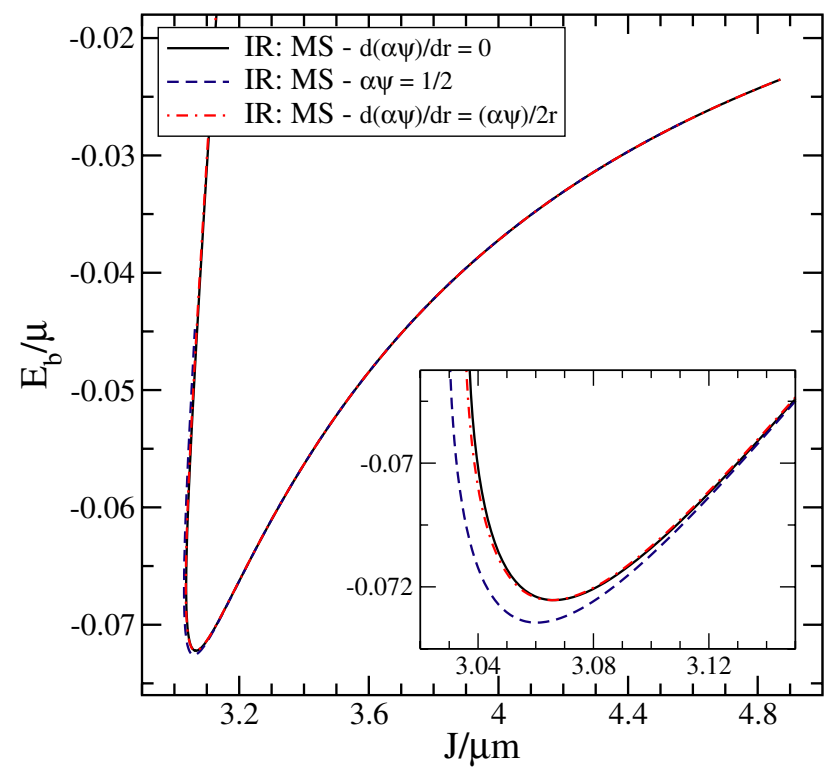

FIG. 15 (color online). Constant $M_{\text {irr }}$ sequence of irrotational equal-mass black holes. Maximal slicing is used in these cases, and three different excision boundary conditions for the lapse are used. 


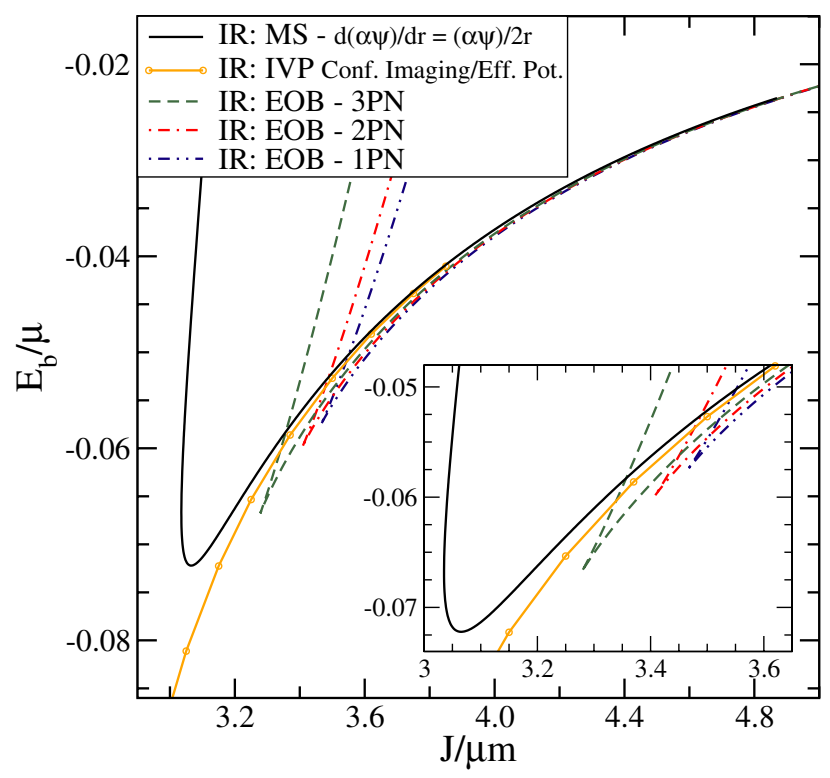

FIG. 16 (color online). Constant $M_{\text {irr }}$ sequence of irrotational equal-mass black holes. Comparison of post-Newtonian EOB sequences with numerical maximal slicing results from Conformal Image and this paper.

conformal-imaging data [39] differ only slightly from the quasiequilibrium results up to the location of the ISCO in the quasiequilibrium sequence. However, the conformalimaging sequence extends to much smaller separations before encountering its ISCO.

Table III displays the ISCO data for irrotational equalmass black holes on a maximal slice. Again, the ISCO is defined in two ways for the QE-sequence data. However, unlike the corotating case, the two definitions disagree dramatically. It is clear that the ISCO, when defined as a minimum of $E_{b}$ along a sequence where $M_{\text {irr }}$ is held fixed, is consistent with the results from the PN data. This is fortunate given the fact that $M_{\mathrm{irr}}$ decreases as the binary separation decreases along sequences where Eq. (75) is satisfied. However, it is unclear why these $E_{\mathrm{ADM}}$ defined ISCOs disagree so significantly from the PN data. The $E_{b}$-defined ISCO data for the various irrotational sequences is plotted with the corotating data in Figs. 10, 12, and 14.

Figs. 10 and 16 plot the binding energy $E_{b}$ as a function of $J$ for the irrotational sequences. Figs. 12 and 17 plot $E_{b}$ as a function of $\Omega_{0}$ for the same set of sequences and for the ISCO. And, Figs. 14 and 18 plot $J$ as a function of $\Omega_{0}$ for the same set of sequences and for the ISCO.

\section{B. Nonmaximal Slicing}

For the case of sequences of corotating or irrotational quasiequilibrium initial data obtained on maximal slices (i.e., $K=0$ ), the choice of the lapse boundary condition seems to have very little effect on gauge-invariant quantities. This lends support to our assertion that the choice

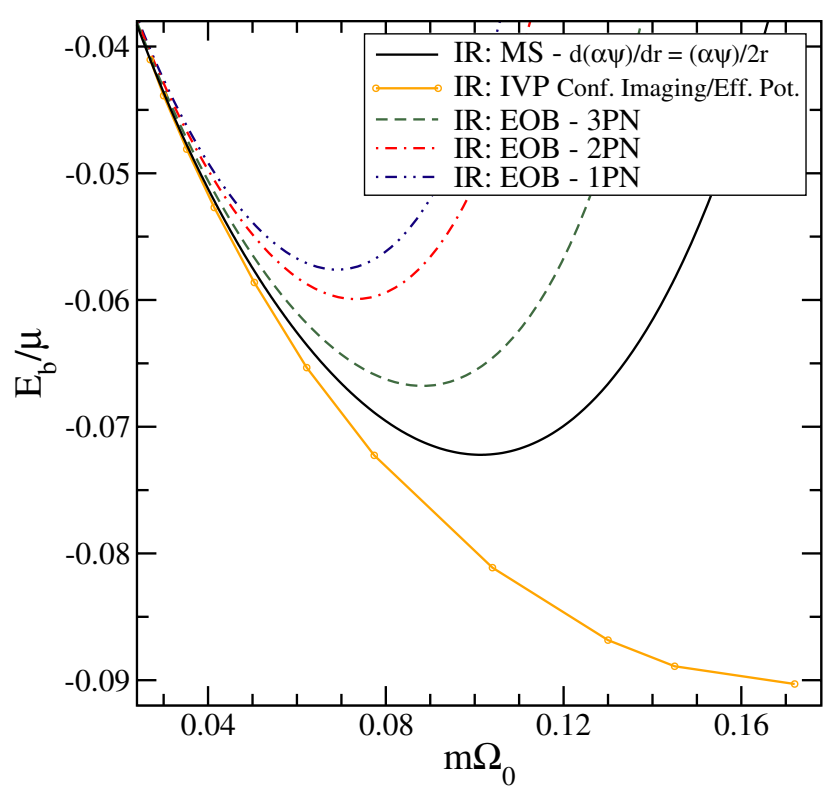

FIG. 17 (color online). Constant $M_{\text {irr }}$ sequence of irrotational equal-mass black holes. Comparison of post-Newtonian EOB sequences with numerical maximal slicing results from Conformal Image and this paper.

of the lapse boundary condition is part of the initial temporal gauge freedom. To further test this assertion, we should consider varying other aspects of the freely specifiable data. Quasiequilibrium considerations demand that we choose $\tilde{u}_{i j}=0$ and $\partial_{t} K=0$. This leaves us the

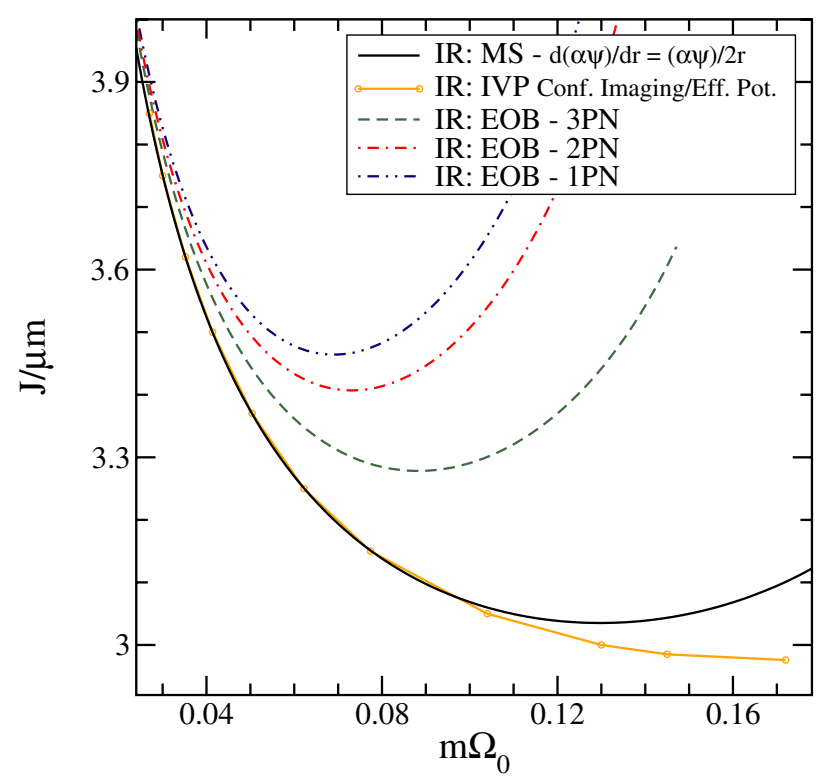

FIG. 18 (color online). Constant $M_{\text {irr }}$ sequence of irrotational equal-mass black holes. Comparison of post-Newtonian EOB sequences with numerical maximal slicing results from Conformal Image and this paper. 
options of choosing a nonflat conformal metric or a nonmaximal slice.

While changing either can affect the content of the dynamical degrees of freedom in the initial data, the conformal metric is more closely tied to these dynamical degrees of freedom and to the spatial gauge freedom. The choice of the trace of the extrinsic curvature is usually thought of as fixing the initial temporal gauge freedom, although as we have seen from the example of the family of maximal slices of Schwarzschild in Sec. IIIC, this is not always sufficient to fix this aspect of the gauge freedom. In any case, it seems reasonable that the best choice is to vary $K$.

A convenient choice for a nonmaximal slicing is one based on a stationary black hole in Kerr-Schild coordinates. For the case of a noncharged, nonspinning black hole these are also referred to as ingoing EddingtonFinkelstein coordinates (cf. Ref. [42]). The spatial metric is given by Eq. (55), and after the coordinate transformation (56) it becomes conformally flat. The trace of the extrinsic curvature for the Kerr-Schild slicing of Schwarzschild is given by Eq. (58c). For a binary system, we use a linear combination of two copies of Eq. (58c), each centered at the location of one of the black holes, to define $K$.

Figs. 19 and 20 display the results for both corotating and irrotational sequences of equal-mass black-hole binaries based on the Kerr-Schild-like slicing described above. In both cases, the same three lapse boundary conditions used for the maximal slicing solutions were again used. When the holes are at large separation, the different lapse boundary conditions cause little variation in the results. However, when the holes are close together, the different lapse boundary conditions cause significant variation in the sequences. From this example it seems that the choice of the lapse boundary condition may have a significant effect on quasiequilibrium solutions of the conformal thin-sandwich equations. However, this example may be somewhat misleading.

Maximal slicing $(K=0)$ is based on a global geometric concept that does not depend on the separation of the black holes in a binary. For an isolated black hole, the Kerr-Schild slicing also has a geometric interpretation. However, a linear combination of the traces of the extrinsic curvatures for individual black holes does not retain this geometrical meaning. Thus, as we vary the separation of the black holes in the nonmaximal slicing sequences, we are also effectively varying the slicing condition. This effect is weak when the holes are at large separation, but becomes significant when the black holes are close together.

In constructing meaningful sequences, everything in the construction of the individual models should be held fixed except for the separation. For maximal slicing, the various choices of the lapse boundary condition choose a

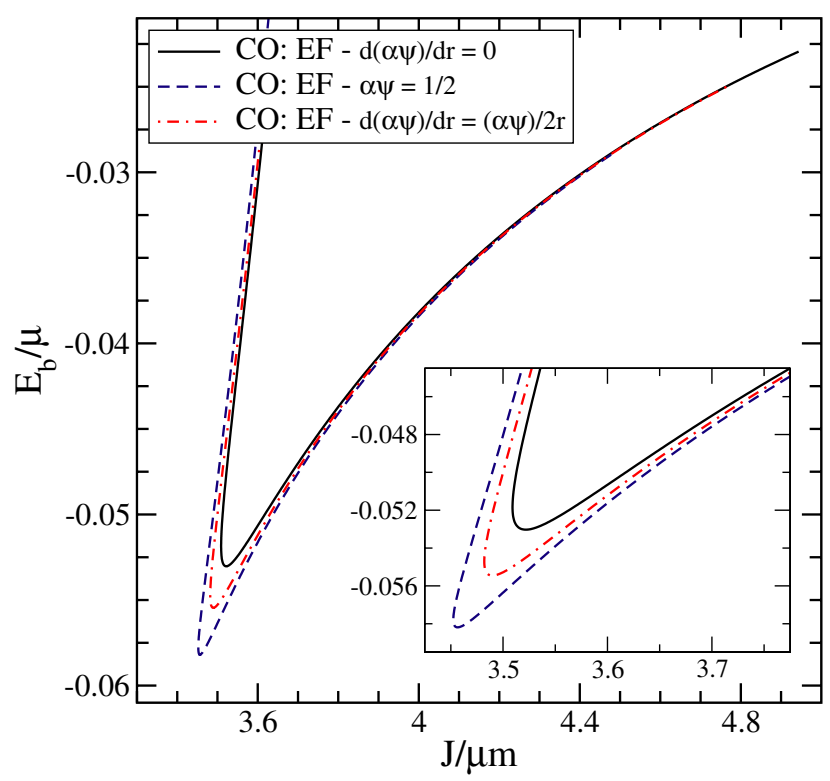

FIG. 19 (color online). Constant $M_{\text {irr }}$ sequence of corotating equal-mass black holes. Eddington-Finkelstein slicing is used in these cases, and three different excision boundary conditions for the lapse are used.

particular slice from among a family of maximal slices. However in the case of the Kerr-Schild-based slicing, it seems likely that the functional form of $K$ as a function of separation and the form of the lapse boundary condition conspire to define a different slicing condition for each

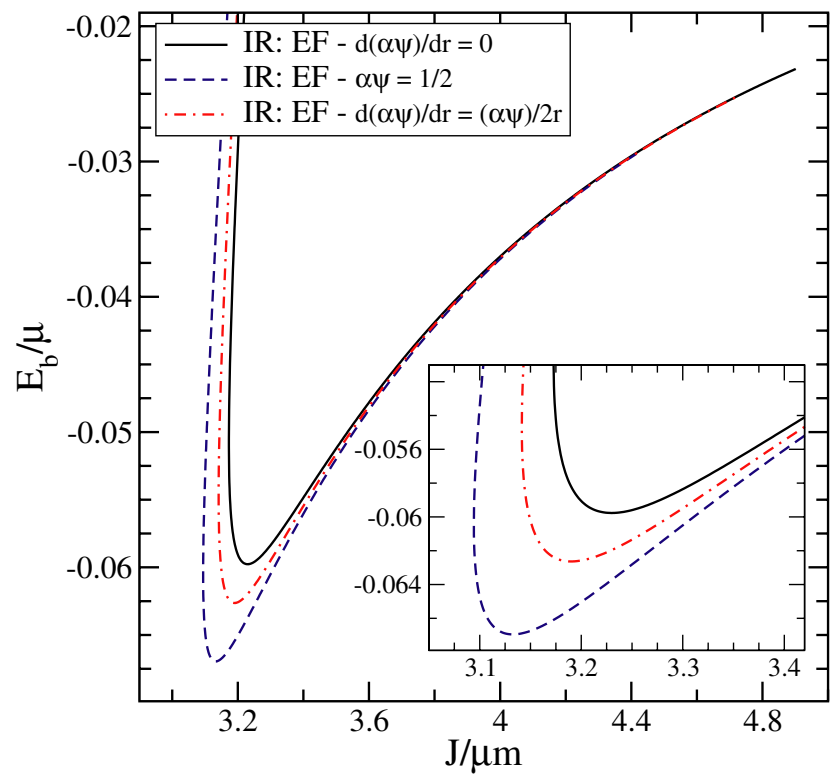

FIG. 20 (color online). Constant $M_{\text {irr }}$ sequence of irrotational equal-mass black holes. Eddington-Finkelstein slicing is used in these cases, and three different excision boundary conditions for the lapse are used. 
initial-data model considered. Therefore, it seems reasonable to question the validity of these nonmaximal slicing sequences. More importantly, we should be cautious in attributing undue significance to the choice of the lapse boundary condition based on this example.

\section{DISCUSSION}

In this paper, we have refined the QE boundary conditions defined originally in Ref. [1] and explored both single and binary black-hole configurations. The original motivation in deriving these boundary conditions was to provide conditions that would be consistent with quasiequilibrium configurations. The binary black hole initialdata sets constructed in Sec. V are intended to be in quasiequilibrium. While the individual black holes in a binary cannot be in true equilibrium, it would be useful to determine if they are roughly in equilibrium.

One measure of this is to see how well the QE lapse condition (51) is satisfied. For stationary black holes, this equation holds. However, in the general case, it does not. Equation (51) defines $\alpha_{\mathrm{BC}}$ as the error in this boundary condition when applied on the excision boundary of one of the holes.

Figure 21 shows both the average value of $\alpha_{\mathrm{BC}}$ and the $L_{2}$ norm of $\alpha_{\mathrm{BC}}$ as a function of $\Omega_{0}$ for a corotating equalmass binary. Figure 22 shows the same information for an irrotational equal-mass binary. At large separations (small $\Omega_{0}$ ), we should expect that each black hole is nearly in equilibrium. For the corotating sequences, $m \Omega_{0} \approx 0.01$ corresponds to a proper separation between the horizons of approximately $20 \mathrm{~m}$. At this separation, $\left|\alpha_{\mathrm{BC}}\right|_{L_{2}} \approx 0.0003$. At the ISCO separation, $m \Omega_{0} \approx 0.11$ corresponding to a proper separation between the horizons of approximately $4.5 \mathrm{~m}$, and $\left|\alpha_{\mathrm{BC}}\right|_{L_{2}}$ has increased by a factor of approximately 20 . As we might expect, the level of violation of the QE lapse boundary condition increases steadily as the separation between the holes decreases. However, there is no dramatic increase in the violation near the ISCO. For the irrotational sequences, $\left|\alpha_{\mathrm{BC}}\right|_{L_{2}}$ begins at $m \Omega_{0} \approx 0.01$ at a level approximately twice as large as that of the corotating sequence. Near the ISCO, it has increased by a factor of approximately 50 .

It seems that the rate of increase in the violation of the QE lapse boundary condition for the irrotational sequences is faster than that seen in the corotating sequences. This is not too surprising when we recall that a true stationary binary configuration can only be achieved for corotating binaries [36]. As with the corotating sequences, the level of violation of the QE lapse boundary condition increases steadily as the separation between the holes decreases, and there is no dramatic increase in the violation near the ISCO.

Another indicator of whether or not each black hole in the binary is in equilibrium is given by the value of $\partial_{t} \psi$ as evaluated on the apparent horizon. We can express the

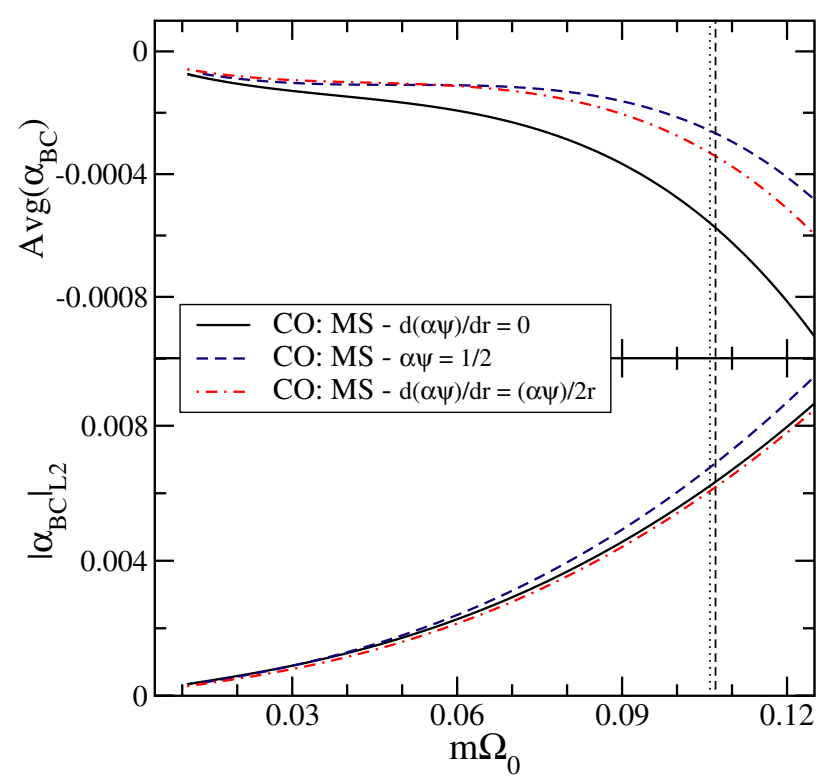

FIG. 21 (color online). Plot of the residual of the quasiequilibrium boundary condition on the lapse (51) of one black hole. Three different choices for the lapse boundary condition are shown for corotating black holes in an equal-mass binary. The upper plot shows the average of the residual over the boundary surface. The lower plot shows the $L_{2}$-norm of the residual. The vertical dashed line shows the approximate location of the ISCO defined by a minimum in $E_{b}$. The vertical dotted line shows the approximate location of the ISCO defined by a minimum in $E_{\mathrm{ADM}}$.

time derivative of the conformal factor on any closed surface as

$$
\begin{aligned}
\partial_{t} \ln \psi= & \frac{1}{4}\left[\tilde{D}_{k} \beta_{\|}^{k}+4 \beta_{\|}^{k} \tilde{D}_{k} \ln \psi\right. \\
& \left.-\frac{1}{2} \tilde{h}_{k \ell} \tilde{u}^{k \ell}+\sqrt{2} \theta-\left(\alpha-\beta_{\perp}\right) H\right] .
\end{aligned}
$$

Clearly, when the QE boundary conditions in Eqs. (17) and (39) are imposed on the excision surface, the last two terms in Eq. (80) vanish. Furthermore, in constructing QE configurations, we have also demanded that $\tilde{u}_{i j}=0$ globally. Therefore, the only terms that are possibly nonzero on the excision surface are those that involve $\beta_{\|}^{i}$.

For corotating binaries, $\beta_{\|}^{i}=0$ and we find that the time derivative of the conformal factor vanishes identically on the excision surface. This is confirmed in our numerical results as shown in the upper half of Fig. 23. There, we see that $\partial_{t} \ln \psi=0$ to roundoff error. For irrotational binaries, the QE conditions require that we take $\beta_{\|}^{i}$ proportional to a conformal Killing vector of $\tilde{h}_{i j}$. This implies that $\beta_{\|}^{i}$ will also be a conformal Killing vector of $h_{i j}$. Unfortunately, the operator acting on $\beta_{\|}$in Eq. (80) is not the conformal Killing operator, but rather 


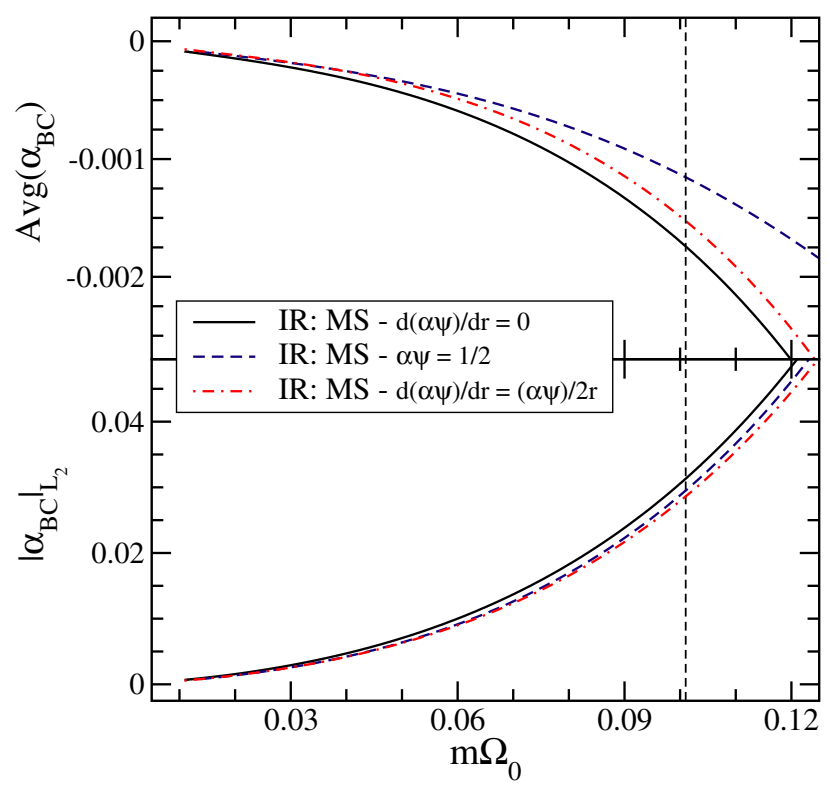

FIG. 22 (color online). Plot of the residual of the quasiequilibrium boundary condition on the lapse (51) of one black hole. Three different choices for the lapse boundary condition are shown for irrotational black holes in an equal-mass binary. The upper plot shows the average of the residual over the boundary surface. The lower plot shows the $L_{2}$-norm of the residual. The vertical dashed line shows the approximate location of the ISCO defined by a minimum in $E_{b}$.

$$
\tilde{D}_{k} \beta_{\|}^{k}+4 \beta_{\|}^{k} \tilde{D}_{k} \ln \psi=D_{k} \beta_{\|}^{k}
$$

While this would vanish if $\beta_{\|}^{k}$ were a Killing vector of $h_{i j}$, it will not vanish if $\beta_{\|}^{k}$ is only a conformal Killing vector of $h_{i j}$, as it will be unless the configuration is truly stationary. Again, this is confirmed in our numerical results where we find that $\partial_{t} \ln \psi \sim 10^{-5}$ when $m \Omega \sim$ 0.01 and grows monotonically as the binary approaches the ISCO. These results are shown in the lower half of Fig. 23.

The quasiequilibrium boundary conditions we have derived and tested in this paper are extremely general. Within the conformal thin-sandwich approach, they will work for any number of black holes that are to be considered in quasiequilibrium, or "isolated." In this paper, we have used several different choices for $K$, but maintained the assumption of conformal flatness. We emphasize that this is not a limitation of the boundary conditions which can, in fact, be used with any viable choice for the conformal three-geometry specified by $\tilde{\gamma}_{i j}$. Furthermore, for binary systems, we have only considered the special cases of corotating and irrotational black holes. Again, this is not a limitation of the boundary conditions which can, in principle, produce any desired spin on the individual black holes.

It has been pointed out that the boundary conditions we have derived are precisely those required to construct a

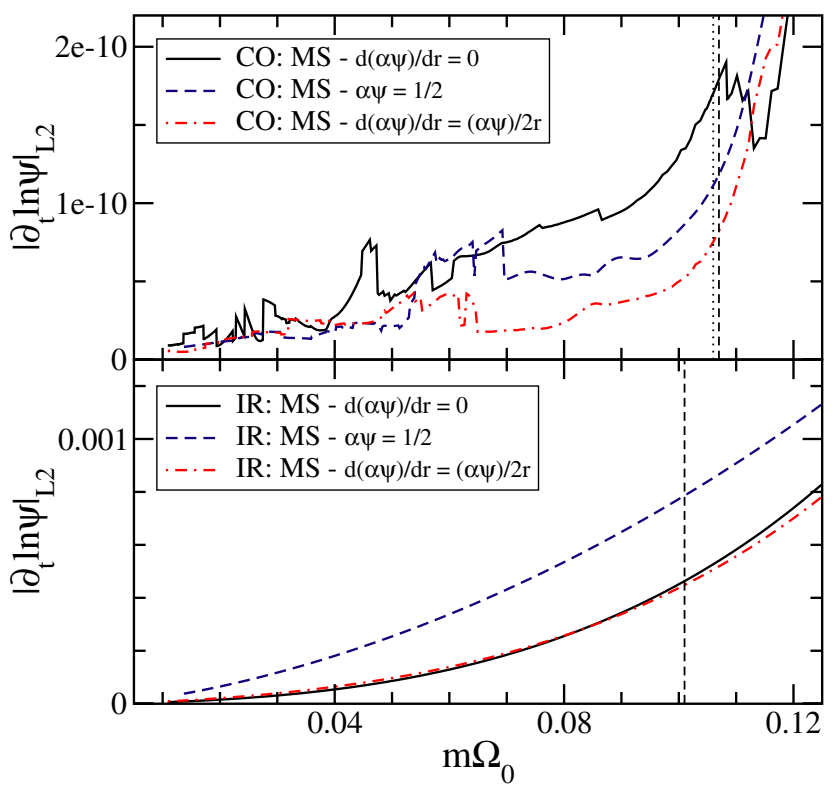

FIG. 23 (color online). Plot of the $L_{2}$-norm of $\partial_{t} \ln \psi$ as evaluated on the excision boundary of one black hole. The upper half of the figure shows the results for corotating black holes in an equal-mass binary, while the lower half shows the results for irrotational black holes. For both cases, three different choices for the lapse boundary condition are shown. In the upper half of the figure, the vertical dashed line shows the approximate location of the ISCO defined by a minimum in $E_{b}$. The vertical dotted line shows the approximate location of the ISCO defined by a minimum in $E_{\mathrm{ADM}}$. In the lower half of the figure, the vertical dashed line shows the approximate location of the ISCO defined by a minimum in $E_{b}$.

black hole satisfying the isolated-horizon conditions [19$21,43]$. This is not surprising since the physical notions underlying an isolated horizon and a black hole in quasiequilibrium are essentially the same thing. It seems likely that the unified approach offered by the isolatedhorizon framework will prove useful in further understanding the physical content of the binary black hole initial-data constructed with the quasiequilibrium boundary conditions and to further understand the role of the lapse boundary condition. In fact, during the final stages of the preparation of this manuscript, we became aware of a paper by Jaramillo et al. [44] that makes the connection between our quasiequilibrium boundary conditions and isolated horizons more precise. This paper argues that the lapse boundary condition, Eq. (51) derived previously in Ref. [1], could be problematic, as we have found and discussed, and shows that weakly isolated horizon considerations do not restrict the lapse boundary condition when constructing initial data, consistent with our findings here. Furthermore, they suggest an alternate boundary condition on the lapse, based on a Lie derivative along the null generators of the horizon. It is not immediately clear that this proposed boundary condition can work. For 
the case of a static black hole, we have shown that essentially any boundary condition on the lapse, when combined with the quasiequilibrium boundary conditions, will yield a valid static slice of Schwarzschild. It may well be that this proposed boundary condition is degenerate similar to Eq. (51) (cf. our discussion in Sec. III C).

Clearly, additional work is required to fully understand the boundary conditions we have derived, and, in particular, the proper role of the lapse boundary condition. However, it is also clear that obtaining appropriate boundary conditions is not the final issue in the quest to construct astrophysically realistic binary black-hole initial data. The most pressing issue is the question of how to make a realistic choice for the conformal three-geometry. While the errors introduced by the assumption of conformal flatness are not "grave," it is clear that we must find a way to allow the physics to dictate the conformal threegeometry instead of choosing it a priori. The approach along these directions outlined in Ref. [45] (see also Ref. [46]) is clearly promising.

\section{ACKNOWLEDGMENTS}

The authors are grateful to J. Isenberg, L. Lindblom, V. Moncrief, N. Ò Murchadha, M. Scheel, M. Shibata, and S. Teukolsky for illuminating discussions. The authors are grateful to K. Thorne and L. Lindblom for their hospitality during the Caltech Visitors Program in the Numerical Simulation of Gravitational Wave Sources in the spring of 2003, during which part of this work was performed. This work was supported in part by NSF Grant No. PHY-0140100 to Wake Forest University, No. PHY9900672 to Cornell University, and No. PHY-0244906

TABLE IV. Sequence of corotating equal-mass black holes on a maximal slice. The length scale is set so that the ADM mass of the binary at infinite separation is one. The ISCO is at separation $d=8.28$.

\begin{tabular}{|c|c|c|c|c|c|c|}
\hline$d$ & $M_{\text {irr }}$ & $E_{\mathrm{ADM}}-1$ & $\Omega_{0}$ & $E_{b}$ & $J_{\mathrm{ADM}}$ & $\ell$ \\
\hline 40 & 0.5000000 & -0.0058296 & 0.01090 & -0.0058296 & 1.2280 & 21.81 \\
\hline 35 & 0.5000001 & -0.0065815 & 0.01327 & -0.0065816 & 1.1655 & 19.17 \\
\hline 30 & 0.5000003 & -0.0075478 & 0.01665 & -0.0075483 & 1.1005 & 16.52 \\
\hline 25 & 0.5000006 & -0.0088277 & 0.02175 & -0.0088289 & 1.0332 & 13.83 \\
\hline 20 & 0.5000013 & -0.0105789 & 0.03012 & -0.0105816 & 0.9647 & 11.11 \\
\hline 19 & 0.5000016 & -0.0110046 & 0.03246 & -0.0110079 & 0.9511 & 10.56 \\
\hline 18 & 0.5000020 & -0.0114600 & 0.03511 & -0.0114639 & 0.9376 & 10.00 \\
\hline 17 & 0.5000024 & -0.0119466 & 0.03814 & -0.0119514 & 0.9243 & 9.444 \\
\hline 16 & 0.5000030 & -0.0124654 & 0.04164 & -0.0124715 & 0.9113 & 8.882 \\
\hline 15 & 0.5000039 & -0.0130163 & 0.04571 & -0.0130240 & 0.8986 & 8.316 \\
\hline 14.5 & 0.5000044 & -0.0133032 & 0.04800 & -0.0133119 & 0.8925 & 8.031 \\
\hline 14 & 0.5000050 & -0.0135969 & 0.05049 & -0.0136068 & 0.8865 & 7.745 \\
\hline 13.5 & 0.5000059 & -0.0138966 & 0.05320 & -0.0139080 & 0.8807 & 7.458 \\
\hline 13 & 0.5000065 & -0.0142009 & 0.05617 & -0.0142140 & 0.8752 & 7.169 \\
\hline 12.5 & 0.5000076 & -0.0145079 & 0.05942 & -0.0145231 & 0.8699 & 6.879 \\
\hline 12 & 0.5000088 & -0.0148151 & 0.06300 & -0.0148328 & 0.8649 & 6.587 \\
\hline 11.5 & 0.5000104 & -0.0151187 & 0.06696 & -0.0151395 & 0.8602 & 6.293 \\
\hline 11 & 0.5000123 & -0.0154139 & 0.07135 & -0.0154385 & 0.8559 & 5.997 \\
\hline 10.5 & 0.5000147 & -0.0156939 & 0.07625 & -0.0157232 & 0.8521 & 5.699 \\
\hline 10 & 0.5000177 & -0.0159493 & 0.08174 & -0.0159847 & 0.8489 & 5.398 \\
\hline 9.5 & 0.5000217 & -0.0161675 & 0.08792 & -0.0162108 & 0.8463 & 5.095 \\
\hline 9 & 0.5000268 & -0.0163311 & 0.09492 & -0.0163846 & 0.8445 & 4.788 \\
\hline 8.9 & 0.5000280 & -0.0163553 & 0.09644 & -0.0164112 & 0.8442 & 4.726 \\
\hline 8.8 & 0.5000293 & -0.0163761 & 0.09799 & -0.0164346 & 0.8440 & 4.665 \\
\hline 8.7 & 0.5000306 & -0.0163934 & 0.09959 & -0.0164546 & 0.8438 & 4.603 \\
\hline 8.6 & 0.5000321 & -0.0164068 & 0.1012 & -0.0164709 & 0.8437 & 4.540 \\
\hline 8.5 & 0.5000336 & -0.0164160 & 0.1029 & -0.0164832 & 0.8436 & 4.478 \\
\hline 8.4 & 0.5000352 & -0.0164209 & 0.1047 & -0.0164913 & 0.8436 & 4.416 \\
\hline 8.35 & 0.5000361 & -0.0164215 & 0.1055 & -0.0164936 & 0.8436 & 4.384 \\
\hline 8.3 & 0.5000369 & -0.0164209 & 0.1064 & -0.0164948 & 0.8436 & 4.353 \\
\hline 8.28 & 0.5000373 & -0.0164203 & 0.1068 & -0.0164949 & 0.8436 & 4.341 \\
\hline 8.2 & 0.5000388 & -0.0164159 & 0.1083 & -0.0164934 & 0.8436 & 4.290 \\
\hline 8.1 & 0.5000407 & -0.0164054 & 0.1102 & -0.0164869 & 0.8437 & 4.227 \\
\hline 8 & 0.5000428 & -0.0163890 & 0.1121 & -0.0164747 & 0.8439 & 4.164 \\
\hline
\end{tabular}


and No. PHY-0099568 to the California Institute of Technology. Computations were performed on the Wake Forest University DEAC Cluster.

\section{APPENDIX A: COROTATING SEQUENCE}

In this Appendix, we list the numerical results for corotating equal-mass black holes assuming conformal flatness, maximal slicing, and using Eq. (59b) for the lapse boundary condition on both excision surfaces. The data has been scaled so that the sequence satisfies Eq. (75) by following the procedure outlined in Eqs. (76)-(79). In order to maintain accuracy in the scaling, the maximum coordinate separation between successive models is $\Delta d=$ 0.05 . Data in the given tables can be easily rescaled to construct sequences with $M_{\text {irr }}$ held constant.
In Table IV, $d$ is the coordinate separation of the centers of the excised regions. $M_{\text {irr }}$ is the irreducible mass associated with one of the black holes. $E_{\mathrm{ADM}}$ is the ADM energy of the system. $\Omega_{0}$ is the orbital angular velocity of the binary system as measured at infinity. $E_{b}$ is the binding energy of the system defined as $E_{b} \equiv E_{\mathrm{ADM}}-$ $2 M_{\text {irr. }} J_{\mathrm{ADM}}$ is the total ADM angular momentum of the binary system as measured at infinity. Finally, $\ell$ is the minimum proper separation between the two excision surfaces as measured on the initial-data slice.

\section{APPENDIX B: IRROTATIONAL SEQUENCE}

In this Appendix, we list the numerical results for irrotational equal-mass black holes assuming conformal flatness, maximal slicing, and using Eq. (59b) for the lapse boundary condition on both excision surfaces. The

TABLE V. Sequence of irrotational equal-mass black holes on a maximal slice. The length scale is set so that the ADM mass of the binary at infinite separation is 1 . The ISCO is at separation $d=8.69$.

\begin{tabular}{|c|c|c|c|c|c|c|}
\hline$d$ & $M_{\text {irr }}$ & $E_{\mathrm{ADM}}-1$ & $\Omega_{0}$ & $E_{b}$ & $J_{\mathrm{ADM}}$ & $\ell$ \\
\hline 40 & 0.5000000 & -0.0058830 & 0.01090 & -0.0058830 & 1.2175 & 21.81 \\
\hline 35 & 0.4999988 & -0.0066618 & 0.01327 & -0.0066594 & 1.1527 & 19.18 \\
\hline 30 & 0.4999963 & -0.0076750 & 0.01665 & -0.0076676 & 1.0846 & 16.52 \\
\hline 25 & 0.4999903 & -0.0090444 & 0.02175 & -0.0090250 & 1.0127 & 13.84 \\
\hline 20 & 0.4999730 & -0.0109891 & 0.03014 & -0.0109351 & 0.9367 & 11.12 \\
\hline 19 & 0.4999661 & -0.0114788 & 0.03247 & -0.0114111 & 0.9211 & 10.57 \\
\hline 18 & 0.4999571 & -0.0120118 & 0.03513 & -0.0119261 & 0.9053 & 10.01 \\
\hline 17 & 0.4999451 & -0.0125938 & 0.03817 & -0.0124839 & 0.8894 & 9.454 \\
\hline 16 & 0.4999286 & -0.0132310 & 0.04169 & -0.0130882 & 0.8734 & 8.893 \\
\hline 15 & 0.4999057 & -0.0139306 & 0.04578 & -0.0137419 & 0.8574 & 8.329 \\
\hline 14.5 & 0.4998908 & -0.0143062 & 0.04808 & -0.0140878 & 0.8494 & 8.045 \\
\hline 14 & 0.4998729 & -0.0147003 & 0.05059 & -0.0144462 & 0.8414 & 7.759 \\
\hline 13.5 & 0.4998513 & -0.0151140 & 0.05332 & -0.0148166 & 0.8334 & 7.473 \\
\hline 13 & 0.4998248 & -0.0155484 & 0.05632 & -0.0151980 & 0.8255 & 7.185 \\
\hline 12.5 & 0.4997922 & -0.0160042 & 0.05961 & -0.0155887 & 0.8176 & 6.895 \\
\hline 12 & 0.4997517 & -0.0164824 & 0.06324 & -0.0159857 & 0.8098 & 6.604 \\
\hline 11.5 & 0.4997006 & -0.0169834 & 0.06726 & -0.0163846 & 0.8022 & 6.311 \\
\hline 11 & 0.4996356 & -0.0175072 & 0.07174 & -0.0167784 & 0.7946 & 6.016 \\
\hline 10.5 & 0.4995517 & -0.0180530 & 0.07676 & -0.0171564 & 0.7873 & 5.718 \\
\hline 10 & 0.4994416 & -0.0186189 & 0.08241 & -0.0175020 & 0.7802 & 5.419 \\
\hline 9.5 & 0.4992944 & -0.0192006 & 0.08882 & -0.0177893 & 0.7733 & 5.116 \\
\hline 9 & 0.4990934 & -0.0197901 & 0.09616 & -0.0179770 & 0.7670 & 4.811 \\
\hline 8.9 & 0.4990447 & -0.0199079 & 0.09776 & -0.0179972 & 0.7658 & 4.749 \\
\hline 8.8 & 0.4989924 & -0.0200252 & 0.09940 & -0.0180101 & 0.7646 & 4.688 \\
\hline 8.69 & 0.4989306 & -0.0201537 & 0.1013 & -0.0180149 & 0.7633 & 4.620 \\
\hline 8.6 & 0.4988763 & -0.0202582 & 0.1029 & -0.0180108 & 0.7623 & 4.564 \\
\hline 8.5 & 0.4988117 & -0.0203733 & 0.1047 & -0.0179966 & 0.7611 & 4.502 \\
\hline 8 & 0.4984034 & -0.0209241 & 0.1146 & -0.0177308 & 0.7561 & 4.189 \\
\hline 7.5 & 0.4977854 & -0.0213930 & 0.1265 & -0.0169638 & 0.7522 & 3.872 \\
\hline 7 & 0.4967902 & -0.0216779 & 0.1411 & -0.0152582 & 0.7500 & 3.548 \\
\hline 6.9 & 0.4965184 & -0.0216959 & 0.1445 & -0.0147327 & 0.7499 & 3.483 \\
\hline 6.86 & 0.4964004 & -0.0216980 & 0.1459 & -0.0144988 & 0.7499 & 3.457 \\
\hline 6.7 & 0.4958660 & -0.0216708 & 0.1518 & -0.0134029 & 0.7501 & 3.351 \\
\hline 6.5 & 0.4950163 & -0.0215319 & 0.1600 & -0.0115644 & 0.7510 & 3.218 \\
\hline
\end{tabular}


data has been scaled so that the sequence satisfies Eq. (75) by following the procedure outlined in Eqs. (76)-(79). In order to maintain accuracy in the scaling, the maximum coordinate separation between successive models is $\Delta d=$ 0.05 . Data in the given tables can be easily rescaled to construct sequences with $M_{\text {irr }}$ held constant.

In Table $\mathrm{V}, d$ is the coordinate separation of the centers of the excised regions. $M_{\text {irr }}$ is the irreducible mass asso- ciated with one of the black holes. $E_{\mathrm{ADM}}$ is the ADM energy of the system. $\Omega_{0}$ is the orbital angular velocity of the binary system as measured at infinity. $E_{b}$ is the binding energy of the system defined as $E_{b} \equiv E_{\mathrm{ADM}}-$ $2 M_{\text {irr }} . J_{\mathrm{ADM}}$ is the total ADM angular momentum of the binary system as measured at infinity. Finally, $\ell$ is the minimum proper separation between the two excision surfaces as measured on the initial-data slice.
[1] G. B. Cook, Phys. Rev. D 65, 084003 (2002).

[2] J.W. York, Jr., Phys. Rev. Lett. 82, 1350 (1999).

[3] H. P. Pfeiffer and J.W. York, Jr., Phys. Rev. D 67, 044022 (2003).

[4] G. B. Cook, Initial Data for Numerical Relativity, http:// www.livingreviews.org/lrr-2000-5.

[5] A. Lichnerowicz, Journal de mathematiques pures et appliquees 23, 37 (1944).

[6] J.W. York, Jr., Phys. Rev. Lett. 26, 1656 (1971).

[7] J.W. York, Jr., Phys. Rev. Lett. 28, 1082 (1972).

[8] G. B. Cook, S. L. Shapiro, and S. A. Teukolsky, Phys. Rev. D 53, 5533 (1996).

[9] E. Gourgoulhon, P. Grandclément, and S. Bonazzola, Phys. Rev. D 65, 044020 (2002).

[10] C.W. Misner, Ann. Phys. (N.Y.) 24, 102 (1963).

[11] R.W. Lindquist, J. Math. Phys. (N.Y.) 4, 938 (1963).

[12] J. M. Bowen and J.W. York, Jr., Phys. Rev. D 21, 2047 (1980).

[13] A. D. Kulkarni, J. Math. Phys. (N.Y.) 25, 1028 (1984).

[14] J.W. York, Jr. and T. Piran, in Spacetime and Geometry, edited by R. A. Matzner and L. C. Shepley (University of Texas, Austin, 1982), pp. 147-176.

[15] M.W. Choptuik and W. G. Unruh, Gen. Relativ. Gravit. 18, 813 (1986).

[16] G. B. Cook, Phys. Rev. D 44, 2983 (1991).

[17] G. B. Cook, M.W. Choptuik, M. R. Dubal, S. Klasky, R. A. Matzner, and S. R. Oliveira, Phys. Rev. D 47, 1471 (1993).

[18] P. Grandclément, E. Gourgoulhon, and S. Bonazzola, Phys. Rev. D 65, 044021 (2002).

[19] A. Ashtekar, C. Beetle, O. Dreyer, S. Fairhurst, B. Krishnan, J. Lewandowski, and J. Wiśniewski, Phys. Rev. Lett. 85, 3564 (2000).

[20] O. Dreyer, B. Krishnan, D. Shoemaker, and E. Schnetter, Phys. Rev. D 67, 024018 (2003).

[21] A. Ashtekar and B. Krishnan, Phys. Rev. D 68, 104030 (2003).

[22] H. P. Pfeiffer, G. B. Cook, and S. A. Teukolsky, Phys. Rev. D 66, 024047 (2002).

[23] B. L. Reinhart, J. Math. Phys. (N.Y.) 14, 719 (1973).
[24] F. Estabrook, H. Wahlquist, S. Christensen, B. DeWitt, L. Smarr, and E. Tsiang, Phys. Rev. D 7, 2814 (1973).

[25] R. Beig and N. O. Murchadha, Phys. Rev. D 57, 4728 (1998).

[26] A. Garat and R. H. Price, Phys. Rev. D 61, 124011 (2000).

[27] J. A. V. Kroon, Phys. Rev. Lett. 92, 041101 (2004).

[28] G. B. Cook, S. L. Shapiro, and S. A. Teukolsky, Astrophys. J. 422, 227 (1994).

[29] R. A. Matzner, M. F. Huq, and D. Shoemaker, Phys. Rev. D 59, 024015 (1999).

[30] P. Marronetti and R. A. Matzner, Phys. Rev. Lett. 85, 5500 (2000).

[31] H.-J. Yo, J. N. Cook, S. L. Shapiro, and T.W. Baumgarte, gr-qc/0406020 [Phys. Rev. D (to be published)].

[32] H. P. Pfeiffer, L. E. Kidder, M. A. Scheel, and S. A. Teukolsky, Comput. Phys. Commun. 152, 253 (2003).

[33] J.W. York, Jr., in Sources of Gravitational Radiation, edited by L. L. Smarr (Cambridge University Press, Cambridge, England, 1979), pp. 83-126.

[34] C.W. Misner, K. S. Thorne, and J. A. Wheeler, Gravitation (W. H. Freeman and Company, New York, New York, 1973).

[35] T. Damour, E. Gourgoulhon, and P. Grandclément, Phys. Rev. D 66, 024007 (2002).

[36] J. L. Friedman, K. Uryū, and M. Shibata, Phys. Rev. D 65, 064035 (2002)

[37] R. Sorkin, Astrophys. J. 249, 254 (1981).

[38] R. D. Sorkin, Astrophys. J. 257, 847 (1982).

[39] G. B. Cook, Phys. Rev. D 50, 5025 (1994).

[40] D. Christodoulou, Phys. Rev. Lett. 25, 1596 (1970).

[41] L. Blanchet, Phys. Rev. D 65, 124009 (2002).

[42] L. E. Kidder, M. A. Scheel, S. A. Teukolsky, E. D. Carlson, and G. B. Cook, Phys. Rev. D 62, 084032 (2000).

[43] A. Ashtekar (private communication).

[44] J. L. Jaramillo, E. Gourgoulhon, and G. A. M. Marugán, gr-qc/0407063.

[45] M. Shibata, K. Uryū, and J. L. Friedman, Phys. Rev. D 70, 044044 (2004).

[46] G. Schäfer and A. Gopakumar, Phys. Rev. D 69, 021501 (2004). 